Article

\title{
Biophysical, Biochemical and Biomedical Evaluation of Colla- gen Hydrolysate in Comparison to Sulfated Glucosamine from Marine Organisms and Lipids of Fish Oil Origin Used as Chon- droprotective Food Supplements ${ }^{\dagger}$
}

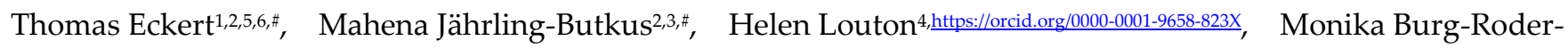
feld ${ }^{2,5}$, Ning Zhang1,7,*, Ruiyan Zhang1,7, Karsten Hesse ${ }^{8}$, Athanasios K. Petridis9, https://orcid.org/0000-0002-3321-5281, Tibor Kožár 10,https://orcid.org/0000-0002-8167-1791, Jürgen Steinmeyer11,https://orcid.org/0000-0002-4053-503X, Roland Schauer ${ }^{12}$, Peter Engelhard ${ }^{1}$, Anna Kozarova13, John W. Hudson 13,https:///rcid.org/0000-0003-3131-5411, Hans-Christian Siebert1,,"https:///rid.org/0000-0001-6282-1777

${ }^{1}$ RI-B-NT - Research Institute of Bioinformatics and Nanotechnology, Schauenburgerstr. 116, 24118 Kiel, Germany; 2Institut für Veterinärphysiolgie und -Biochemie, Fachbereich Veterinärmedizin, Justus-Liebig-Universität Gießen, Frankfurter Str. 100, 35392 Gießen, Germany.

${ }^{3}$ Tierarztpraxis Dr. Silke Fritscher, Bergstraße 104, 73441 Bopfingen-Flochberg, Germany.

${ }^{4}$ Animal Health and Animal Welfare, Faculty of Agricultural and Environmental Sciences, University of Rostock, Justus-von-Liebig-Weg 6b, 18059 Rostock, Germany.

${ }^{5}$ Department of Chemistry and Biology, University of Applied Sciences Fresenius, Limburger Str. 2, 65510 Idstein, Germany.

${ }_{6}^{6}$ RISCC - Research Institute for Scientific Computing and Consulting, Ludwig-Schunk-Str. 15, 35452 Heuchelheim, Germany.

7Institute of BioPharmaceutical Research, Liaocheng University, Liaocheng 252059, China.

${ }^{8}$ Tierarztpraxis Dr. Karsten Hesse, Rathausstraße 16, 35460 Staufenberg, Germany.

${ }^{9}$ Neurochirurgische Klinik, Universität Düsseldorf, Geb. 11.54, Moorenstraße 5, 40255 Düsseldorf, Germany.

${ }^{10}$ Center for Interdisciplinary Biosciences, Technology and Innovation Park, P. J. Safarik University, Jesenna 5, 04001 Kosice, Slovakia.

${ }^{11}$ Laboratory for Experimental Orthopaedics, Department of Orthopaedics, Justus Liebig University Giessen, Paul-Meimberg-Str. 3, 35392 Giessen, Germany.

${ }^{12}$ Biochemisches Institut, Christian-Albrechts Universität Kiel, Olshausenstrasse 40, Kiel 24098, Germany.

${ }^{13}$ Department of Biomedical Sciences, University of Windsor, Windsor, Ontario, Canada, N9B 3P4

E-mails: ${ }^{1,25,6}$ Thomas.Eckert@vetmed.uni-giessen.de, ${ }^{2,3}$ mahenabutkus0@gmail.com,

4helen.louton@uni-rostock.de, ${ }^{2,5}$ monika.burg-roderfeld@hs-fresenius.de,

1,7 zhangning1111@126.com, 1,7zry147896@163.com, ${ }^{8}$ k.hesse@tiermedizin-drhesse.de,

${ }^{9}$ opticdisc@aol.com, ${ }^{10}$ ktibor@rogers.com

11

13jhudson@uwindsor.ca, ${ }^{1}$ hcsiebert@aol.com

+We dedicate this article to the memory of our colleague Roland Schauer who passed away on October 24, 2019

\#contributed equally 
*Correspondence: Hans-Christian Siebert: hcsiebert@aol.com;

Ning Zhang: zhangning1111@126.com

Keywords: osteoarthritis, collagen-hydrolysate, sulfated N-acetyl glucosamine, sialic acids, eicosapentaenoic acid (EPA), MMP-3, ADAMTS-5

Abstract: The bioactivities of collagen-hydrolysates, sulfated glucosamine and a special fatty acid enriched dog-food were tested in a dog patient study as potential therapeutic treatment options in early osteoarthritis. Biophysical, biochemical, cell biological and molecular modeling methods support that these well-defined substances may act as effective nutraceuticals. Importantly, the applied collagen-hydrolysates as well as sulfated glucosamine residues from marine organisms were strongly supported by both an animal model and molecular modeling of intermolecular interactions. Molecular modeling of predicted interaction dynamics were evaluated for the receptor proteins MMP-3 and ADAMTS-5. These proteins play a prominent role in the maintenance of cartilage health as well as innate and adapted immunity. Nutraceuticals data were generated in a veterinary clinical study focusing on mobility and agility. Specifically, key clinical parameters were obtained from blood probes of German shepherd dogs with early osteoarthritis symptoms fed with collagenhydrolysates or sulfated glucosamines. Collagen-hydrolysate, a chondroprotective food supplement was examined by high resolution NMR experiments. Molecular modeling simulations were used to further characterize the interaction potency of collagen-fragments and glucosamines with protein receptor structures. Potential beneficial effects of collagen-hydrolysates, sulfated glycans (i.e. sulfated glucosamine from crabs and mussels) and lipids, especially, eicosapentaenoic acid (extracted from fish oil) on biochemical and physiological processes are discussed here in the context of human and veterinary medicine.

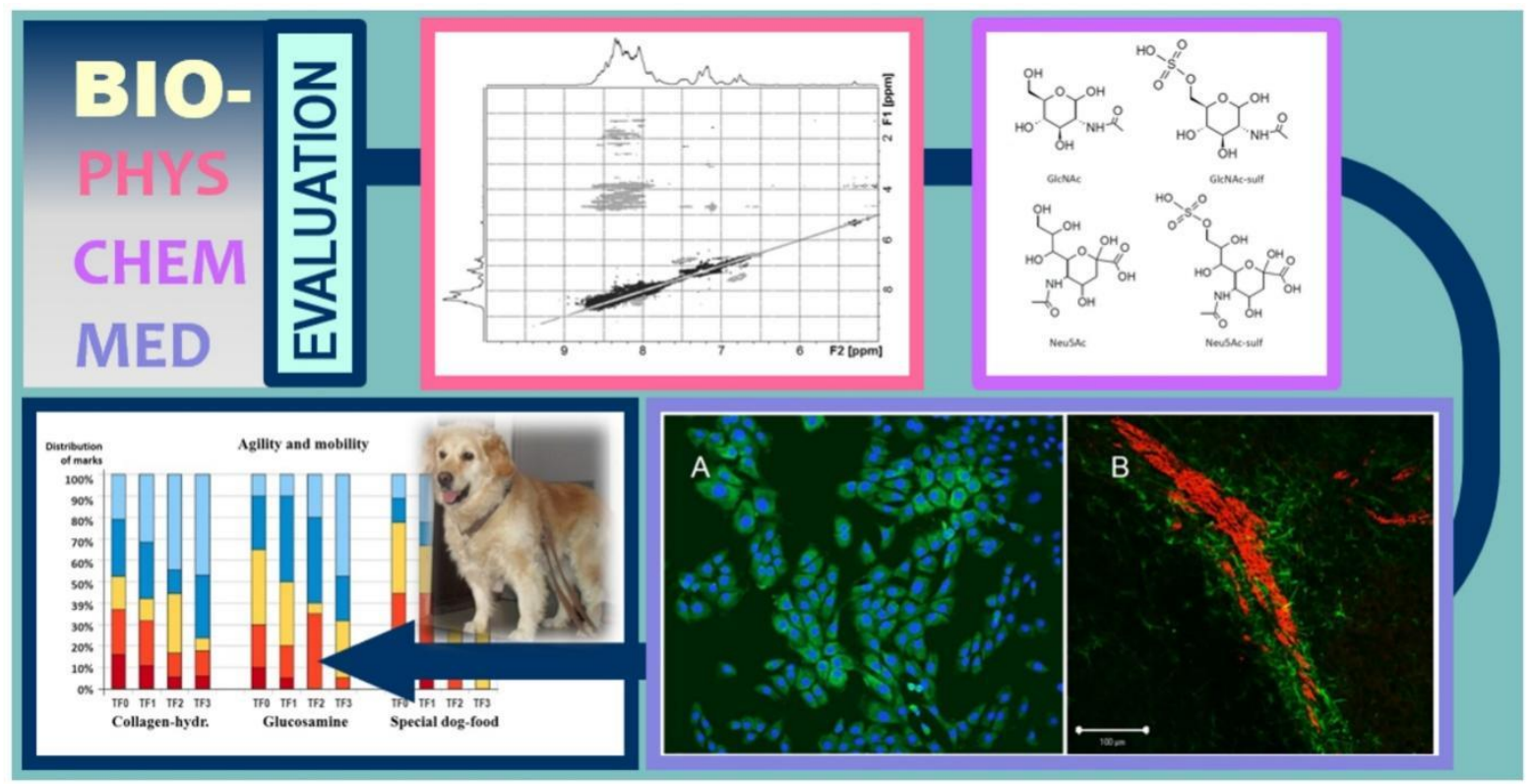

Graphinal Abstract

\section{Introduction}


Profound knowledge about the composition and the molecular structure of the applied substances [1-11] is an essential prerequisite for a reasonable prophylactic cartilage therapy with food supplements (nutraceuticals) in respect to joint health of mammals. Furthermore, detailed data regarding the impact of these food supplements on cartilage health of the patients under study are necessary. This was evaluated in a veterinary clinical study focusing on the mobility and agility of the patients as well as on clinical parameters obtained from blood probes. Possible beneficial effects of collagen-hydrolysates, sulfated glycans (e. g. sulfated glucosamine) and lipids on biochemical and physiological processes within the extracellular matrix of mammalian cartilage tissues are discussed in the context of human- and veterinary-medicine. Thereby, we have explored potential therapeutic effects in our clinical study on various dogs. Dogs with early osteoarthritis (OA) symptoms were fed with collagen-hydrolysate, sulfated glucosamine or a lipid- and vitamin-enriched dog food. In addition to our external observational assessment on the therapeutic value of these compounds we also sought to further dissect the mechanism involved in these effects.

Beneficial effects of collagen- and proteoglycan-fragments may be related to specific interactions with receptors (e.g. integrins or ACEII receptors - in the case of collagen-hydrolysates) and aggrecanases (e.g. ADAMTS-5) or other matrix metalloproteinases (e.g. MMP-3, TIMP-1) [12-15]. Beside these specific interactions unspecific contacts between collagen-strands [16-20] proteoglycans and fatty acids within the extracellular matrix of the cartilage may also play a crucial role when explaining the therapeutic effects on a sub-molecular size level. Therefore, we assessed the effect of the applied substances on the biology the animals by a combination of biophysical, biochemical, cell-biological methods and molecular modeling studies. Similar methodologies have previously been experimentally used to assess the effect of these and other biologically related molecules [9,10,21-29]. For example, the collagen-hydrolysate [30-35] under study has been examined with high resolution NMR experiments. Additionally, molecular modeling approaches such as molecular docking and molecular dynamics simulations have been carried out in order to obtain more information about the interaction potency of collagen-fragments and sulfated GlcNAc with receptor structures. As a result we are now able to formulate efficient encapsulation strategies [36-38] for an oral application of peptides and proteins $[39,40]$.

The concept of this study was to provide a comparative examination of the potential benefits of nutraceuticals as chondroprotective agents. Thereby we tested a specific collagen-hydrolysate, sulfated glucosamine from marine organisms as well as fish oil lipids, and vitamin enriched dog food.

Molecular modeling studies of the applied nutraceuticals are essential since they provide valuable hints on how these substances could influence biochemical processes not only in dogs and horses but also in humans. Former studies illustrate the problems of carbohydrates and glycans structural evaluation [41]. Molecular docking studies of carbohydrates are still under development [42] selected modeling examples are well documented for glycomimetic peptides and sulfated saccharides [21] as well as with linear collagen-fragments $[1,4,10]$. Furthermore, polysialic acid (polySia), polySia mimetics and sulfated polysaccharides have been analyzed by us this way [21,24].

The presence/absence of sulfate groups on glycans has an impact on receptor interactions in addition to having an effect on triple-helical collagen-strands [43]. These variations are caused by direct interactions between the proteoglycan and the triple-helical collagen-strand. However, the interaction process can also be triggered by conformational differences within the carbohydrate chains of the proteoglycans [9] leading to an 
indirect influence in respect to the molecular recognition process. Beside unspecific interactions of proteoglycan fragments with collagen-strands, it is possible to discuss a potential impact on enzymatic activity caused by collagen-fragments or glucosamine. Here, aggrecanase and other MMPs have been in silico studied with help of structural data sets [12-15] available in PDB. The impact of the presence and the absence of a sulfategroup on saccharides was described previously in quantum chemical detail [10]. It turned out that the presence of a sulfate group alters the orbital structure of non-sulfated GlcNAc and GalNAc residues in a significant way. This could indeed influence specific and unspecific interactions with various target structures [10]. The effects of the high-quality dog-food in the control group are discussed in light of recently published biochemical and physiological data on lipids of interest $[39,40]$.

The current approach described here was to utilize a combination of bioinformatic and molecular modeling tools, biochemical and biophysical analysis with cell biological and clinical studies to make it possible to evaluate the therapeutic impact of bio-active components of collagen-hydrolysates and proteoglycans.

\section{Results}

\subsection{Drug Administration and Dog Studies.}

Various dogs with early osteoarthritis (OA) were examined in a randomized clinical study examining the therapeutic effect of nutraceuticals. Specifically, the impact of A) a collagen-hydrolysate, B) tablets of sulfated glucosamine and C) a high-quality dog-food i.e. Hills-JD (containing vitamins and enriched with fatty acids, especially EPA) on joint health was evaluated. We sought to conduct a comparative analysis of the potential benefits of these compounds and to determine the molecular interactions between collagen-fragments, parts of the glycan chains and lipids in the extracellular matrix, in order to provide novel information about the biochemical mechanisms which underlie the maintenance of mammalian cartilage tissue. The dogs were fed Hills-JD instead of a placebo, since the dogs already displayed osteoarthritis symptoms and were therefore already being treated accordingly. The main lipid-component of HillsJD is eicosapentaenoic acid (EPA) which is enriched in sea-fishes such as salmon and herring. The administered peptides, carbohydrates and lipids were delivered via the gastro-intestinal tract into the blood-stream and in this way to the crucial target tissues in the organism. A dose of $20 \mathrm{~g}$ collagen-hydrolysate was administered per day and dog patient (based on the daily collagen requirements in food for wolves). The dose was not reduced for smaller dogs since a higher amount of this nutraceutical is completely harmless (as tested in cell biological assays). For comparison we previously analyzed the effect of collagen-hydrolysate on selected horses $(50 \mathrm{~g} /$ day $)$ and a Shetland pony ( $25 \mathrm{~g} /$ day) that displayed arthritic related problems in their movements. Different horse races were examined: Holstein horse, Hanoverian horse, Arabian horse, American Quarter horse, Trotter, English Blood horse and Trakehner horse. Our current observations are in full agreement with a dog- and a horse-study recently published [32,33].

The impact of these nutritional supplements on the mobility of randomly selected dogs of the study (Table 1) was evaluated and documented by video at both the beginning and end of the therapy. Representative videos for two patients from the sulfated glucosamine group, two from the collagen-hydrolysate group and one dog from the control group are available for viewing at the following link:

https://www.dropbox.com/s/bpu7tcgmz5v41zv/Dog\%20Study\%20Exam\%202021.mp4?dl=0

https://www.dropbox.com/s/bpu7tcgmz5v41zv/Dog\%20Study\%20Exam\%202021.mp4?dl=0.

TABLE 1. Characteristics of randomly selected dogs shown in video presentation 


\begin{tabular}{lllll}
\hline Dog Name & Treatment & Breed & Age [years] & Weight [kg] \\
\hline Buddy & glucosamine & Schnauzer-mix & 5 & 26.5 \\
Cora & glucosamine & Newfoundland dog & 7 & 54 \\
Sarabi & collagen-hydrolysate & Boerboel & 3 & 45 \\
Tobi & collagen-hydrolysate & Half-breed & 8 & 16 \\
Emma & special dog-food with & German shepherd-mix & 7 & 35 \\
& lipids and vitamins & & & \\
Paula & collagen-glucosamine- & Golden Retriever & 12 & 30 \\
& lipid mix & & & \\
\hline
\end{tabular}

Dog-patient handlers were able to easily perceive that dogs in the early stages of OA already exhibited difficulty with stair climbing. The videos clearly display this observation and demonstrate that sixteen weeks of therapy had a positive effect on the ability of these dogs to climb stairs along with no new outwardly discernable negative aspects or progression of disease.

Overall, more than 80 dogs were treated during the 16 weeks therapy period with improvements in agility found in all three groups. To assess pain, one of the symptoms of OA, and any therapeutic progress, palpation was performed independently for the left and right femoral joint throughout the study. Notably, a reduction in tenderness/pain was observed as early as four weeks into treatment (Figure 1). After 16 weeks (the end of

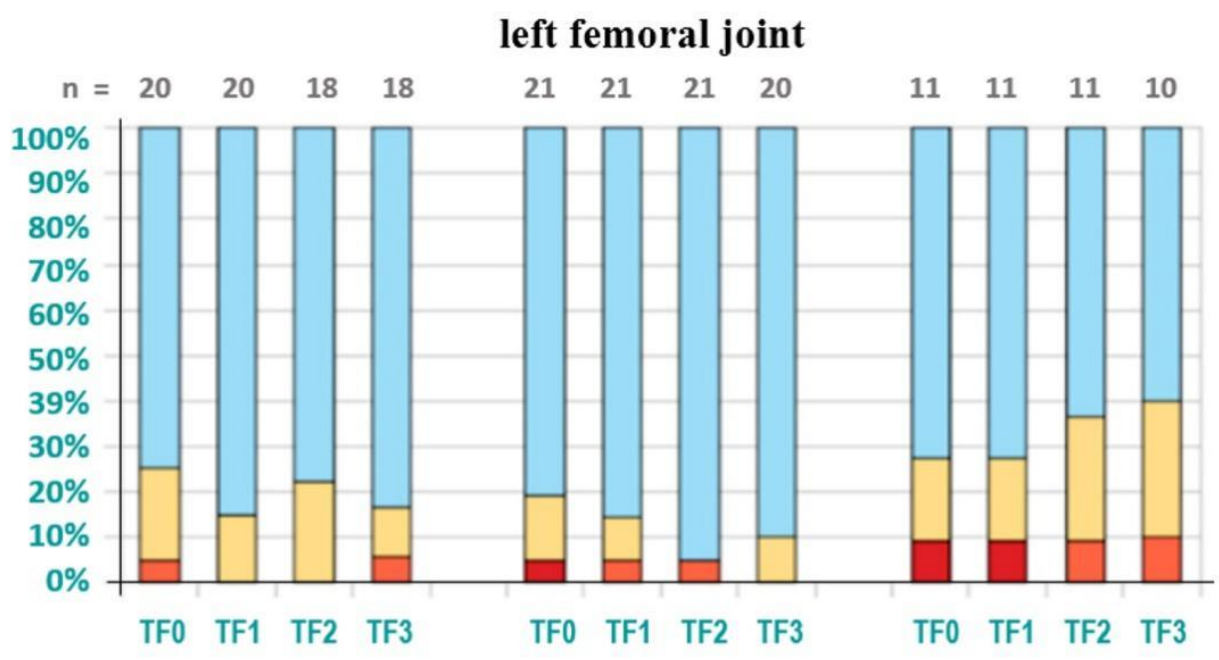

right femoral joint

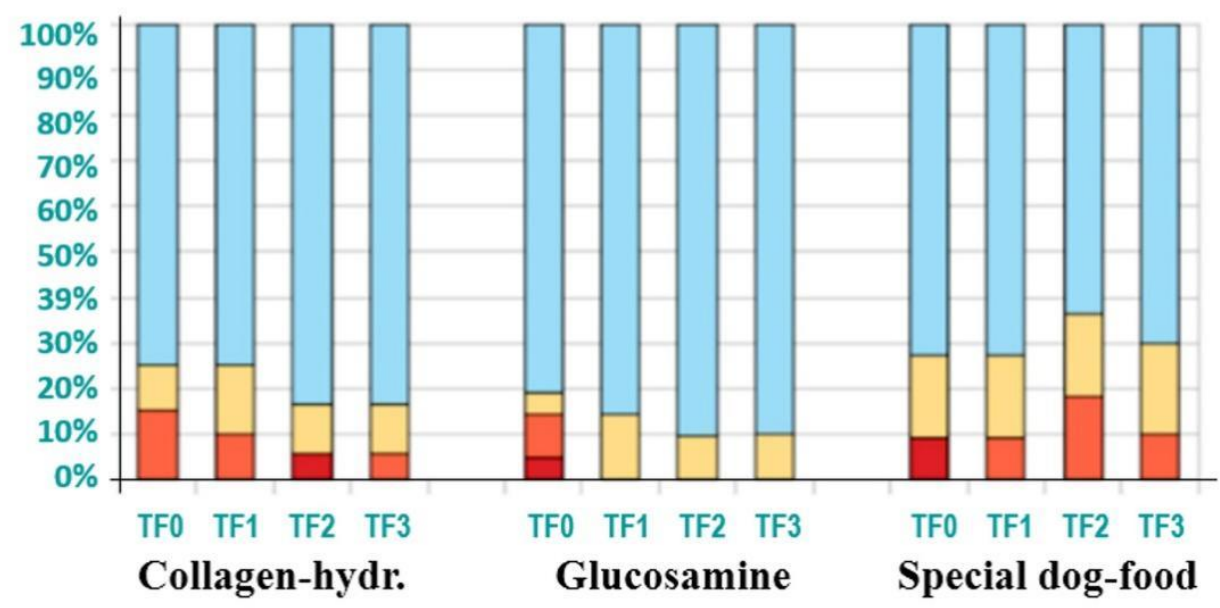


the therapy), all groups (including the control group which contains only the half number of patients) exhibited a significant reduction in the sensitivity of their femoral joints to manipulation.

Figure 1. Canine dog patients with beginning OA symptoms were examined at the different time frames: at TF0 - beginning, TF1 - after 4 weeks, TF2 - after 8 weeks and TF3 - after 16 weeks concerning pain in their left or right femoral joint. Pain symptoms during palpation were examined for all dogs in the three groups according to a clinical standard protocol. Four scores are given: no pain reaction (light blue), minor pain reaction (yellow), stronger pain reaction (orange), strongest pain reaction (red) of QOL. The distribution of the dog patients is shown in percent, $\mathrm{n}$ is the number of dogs examined.

A key indicator of therapeutic value in any trial is the effect of the substances under study on the quality of life (QOL). We therefore assessed QOL using previously published guidelines [44,45]. The QOL-score combines the mood of the animal, its mobility and agility, joy of playing, sounds of pain and problems with climbing stairs. Dog-patient handlers were provided with a questionnaire which evaluated the QOL score (Figure 2). In addition, to remove any perceived bias, a veterinary doctor, not involved in the study in any other capacity, assessed video footage of the treated animals to provide an independent QOL estimate. All substances under study (collagen-hydrolysate, sulfated glucosamine as well as the special dog food enriched with fatty acids and vitamins) resulted in positive effects on QOL score. Collagen-hydrolysate led to the most promising results in terms of displaying moderate to minor symptoms or no joint problems at the focus of study.

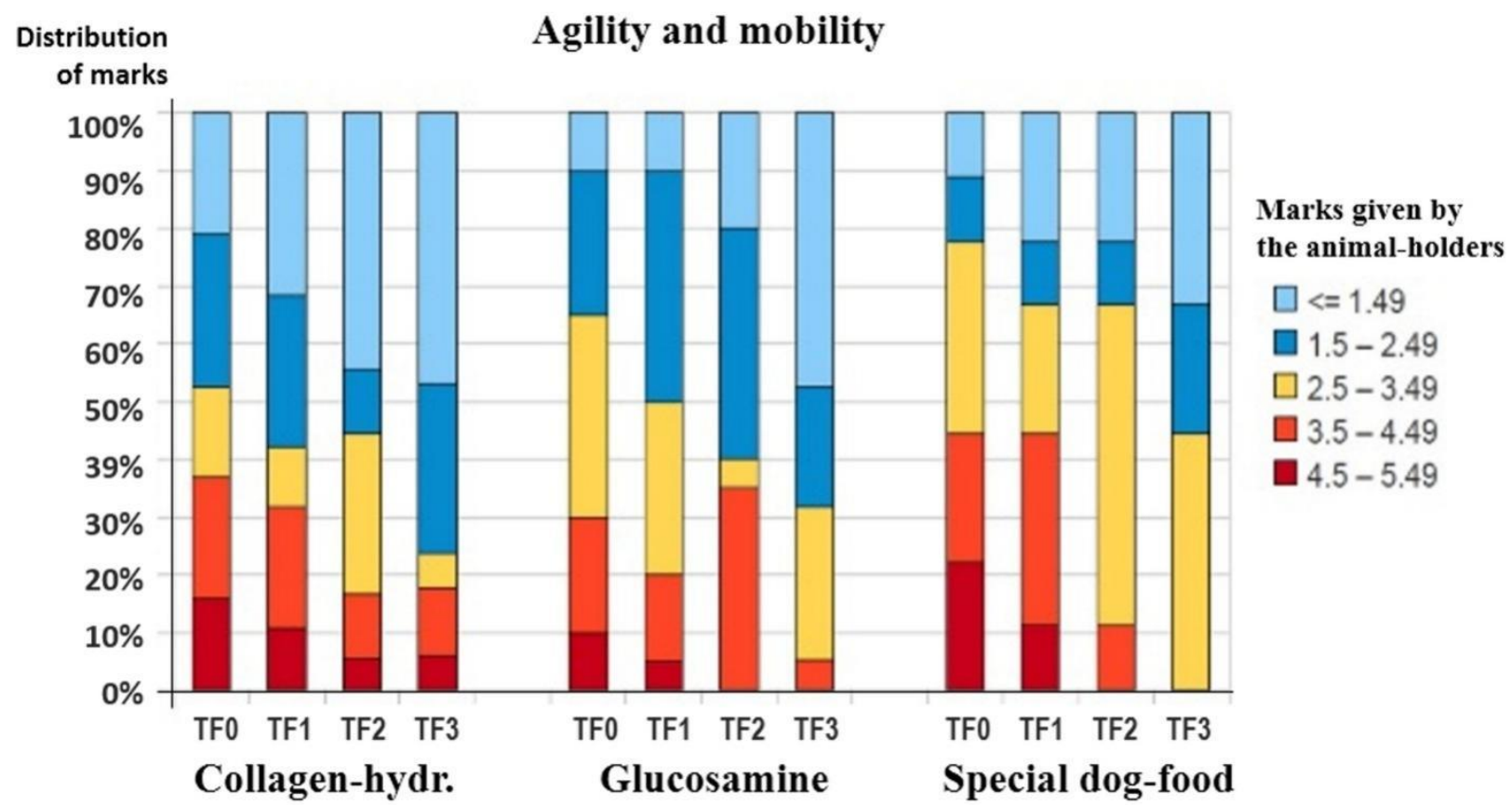

Figure 2. Agility and mobility of dogs with beginning OA symptoms (1: no problems, 2: minor problems, 3: moderate problems, 4: worse problems, 5: extremely large problems) given by the 
animal-holders at different time-points (TF0: at the beginning, TF1: after 4 weeks, TF2: after 8 weeks, TF3: after 16 weeks). These marks correlate with QOL.

Our observations suggest that the collagen-hydrolysate applied in this dog-study contains bio-active fragments that have a beneficial effect on OA symptoms, similar to that observed for sulfated glucosamine. Cellular studies show that the collagen-fragments in the hydrolysate are responsible for the effects within the extracellular matrix of the joint tissue. These effects can be supportive, non-supportive or even detrimental $[5,7,8]$. In order to establish the correlation between structure and function of bio-active components of the collagen-hydrolysate applied in our study (Fortigel from Gelita) we further characterized the relationship by well-established protocols $[1,4,5,7,8]$.

\subsection{Cell Biological Tests.}

The differentiation of canine as well as of equine chondrocytes were studied in the absence and in the presence of collagen-hydrolysates and proteoglycan fragments. The distinct time-dependent differentiation pattern (e. g. in respect to the known sialic acid galactose linkage at the end of the saccharide chains of the corresponding glycoproteins is well established [22] and can be used to test the respective impact of various substances in cell culture very precisely [8]. A representative image displaying the expected pattern for equine chondrocytes grown on collagen is presented in Figure 3A.
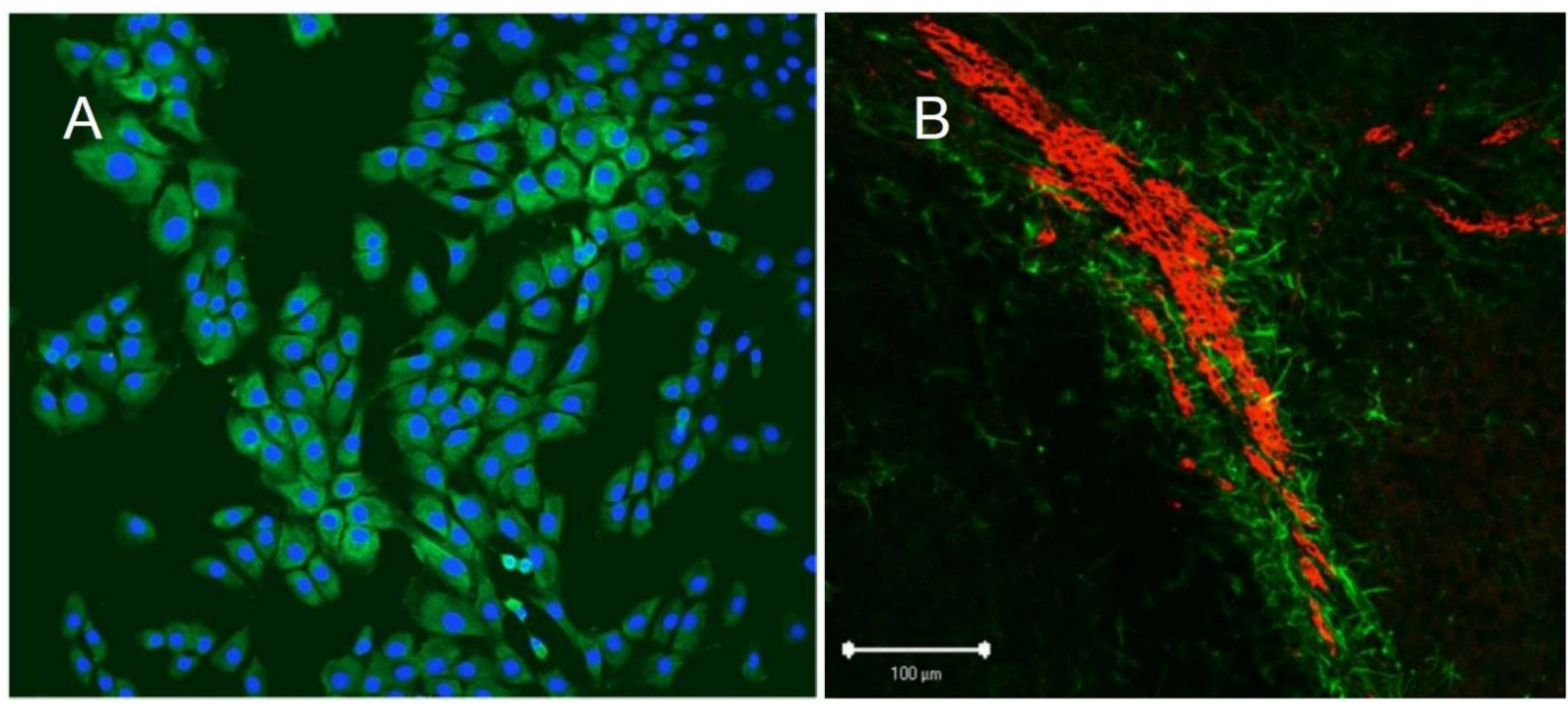

Figure 3. A) Equine chondrocytes are grown on collagen media. The cell nuclei are highlighted by a DAPI staining. The growing collagen-strands around the nuclei are stained in light green. This cell assay was performed as a test-system to determine whether the collagen-hydrolysates resulted in any biological effects and/or potential toxicity. B) The polysialic acid molecules on stem cells are colored in red. The glia cells are colored in dark green. This cell assay can act as suited test-system to control the sialic acid - dependent impact of collagenfragments on cell-differentiation.

The induction of multi-directional differentiation processes of equine and canine chondrocytes strongly depends on the kind of collagen-hydrolysate under study. Furthermore, a stem cell assay can act as suited testsystems to control the sialic acid - dependent uptake of collagen-fragments $[22,46]$. Shown if Figure $3 \mathrm{~B}$, is an 
example of a stem assay which depicts the effect of sialic acid on the differentiation of glia. In addition, our cell biological studies demonstrate that the collagen-hydrolysate applied in this dog study has no toxic effects.

\subsection{Blood Parameters.}

Blood parameters were obtained from a homogenous group of dog patients (23 German Shepherd dogs) in which collagen-hydrolysate was tested as a food supplement. Previous studies have focused on a correlation between MMP-3 plasma levels and MMP-3 synovia levels for dogs suffering from osteoarthritis [44,45]. Since MMP-3 is a highly proteolytic enzyme, enhanced breakdown of cartilage tissue in the German shepherd dog OA group could occur via degradation of collagen types II, IX, X [47] and aggrecan [48]. Additionally, TIMPs are known inhibitors of MMP within tissues. We therefore examined MMP and TIMP levels at various time points throughout the study. Notably, MMP-3 levels were significantly reduced $(p \leq 0.05)$ after 8 weeks of treatment. We did not find a significant alteration in TIMP-1 levels during this same time period (data not shown). Our results suggest that like sulfated and non-sulfated glucosamines, small collagen-fragments may have an influence on the activity of matrix metalloproteinases. Given this result, allosteric inhibition and stimulation as well as competitive inhibition were considered as additional mechanism to be investigated when using collagen-hydrolysates or proteoglycan fragments i.e. sulfated and non-sulfated glucosamines as nutraceuticals.

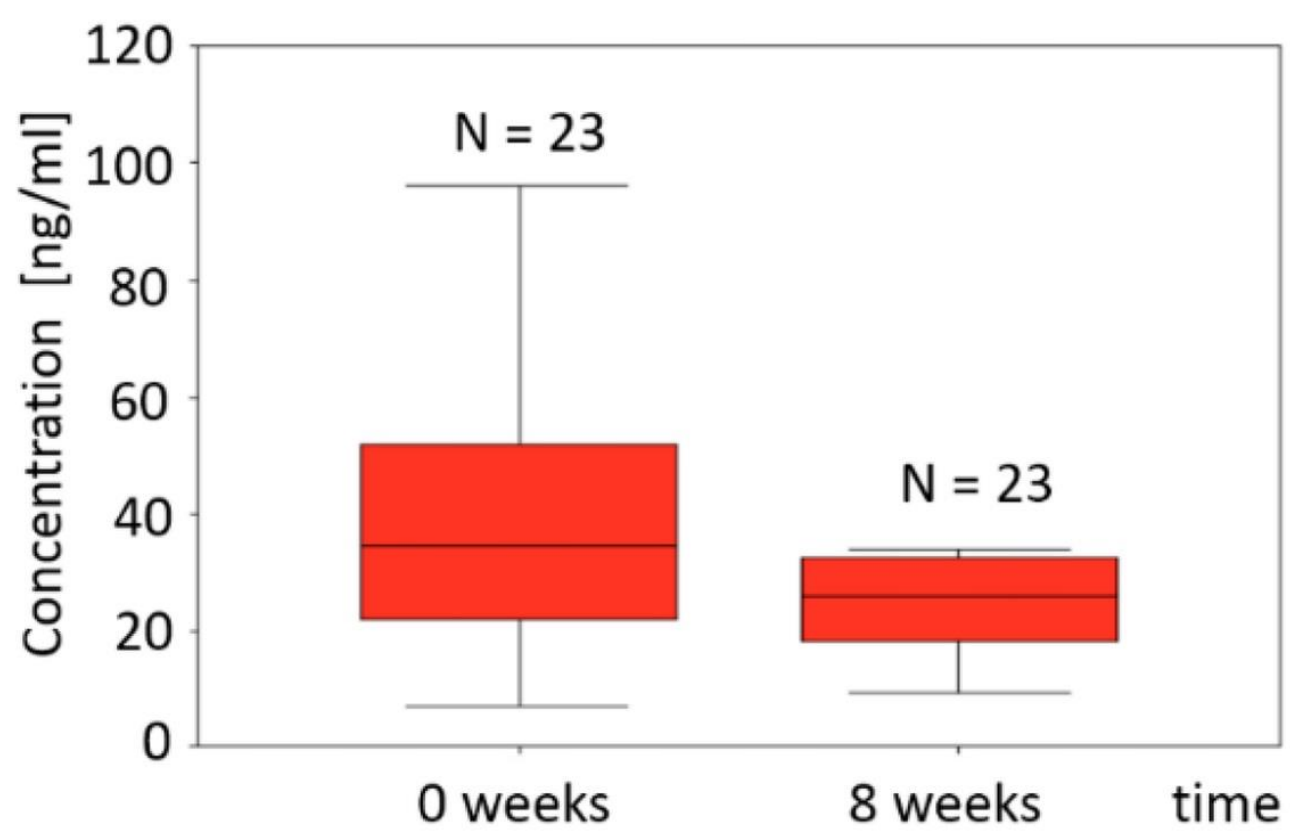

Figure 4. MMP-3 levels in German shepherd dogs with early OA in which their diet was supplemented with collagen-fragments. The concentration values at the y-axis correspond to $\mathrm{ng} / \mathrm{ml}$. Shown are the levels at the beginning and after 8 weeks of treatment.

2.4. NMR Analysis of Fortigel Collagen-hydrolysate. 
Our studies indicated that pain reducing effects are detectable by an evaluation of the mobility and agility of the animals under study. Furthermore, biochemical parameters are also altered as found in our analysis of the blood-probes in respect to osteoarthritis markers (e.g. TIMP-1, MMP-3). These observations are probably related to the occurrence of certain bio-active collagen-fragments within the hydrolysates. It was therefore of interest to determine whether the positive effect on cartilage health for dog patients with beginning

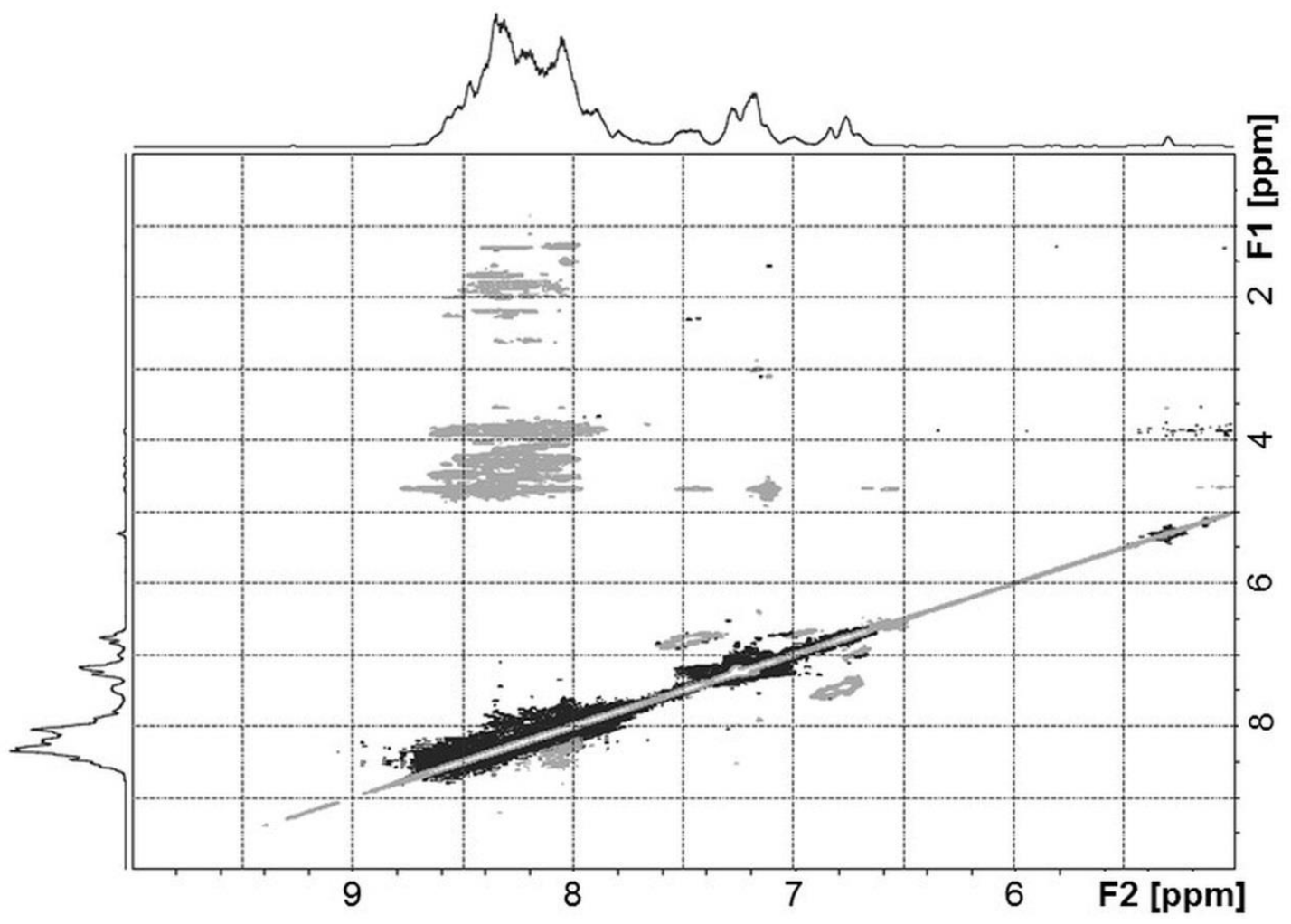

osteoarthritis symptoms can be correlated with the applied collagen-fragment mixture. We therefore conducted DOSY NMR experiments as described previously [4]. Our NMR results indicate that the size-range of the collagen-fragments in the collagen-hydrolysate food supplement is between $2.9-8.1 \mathrm{kDa}$ (Figure 5), with no triple helical collagen structures present [1]. We note that mobility and agility are not automatically related to cartilage health. They might depend on a placebo effect or a general pain reduction as well as physiological improvements. Since the collagen-hydrolysates differed in their composition we further characterized them to provide a more detailed molecular analysis. Mass-spectrometry and NMR have provided previously a detailed molecular analysis of the collagen-hydrolysates under study $[1,4,5,7]$. In the present study, we used TOCSY to identify specific amino acid residues (e. g. Arg residues) of these bio-active compounds (Figure 6). This type of analysis has allowed to characterize the collagen-hydrolysate unambiguously.

Figure 5. DOSY NMR spectrum of the collagen-hydrolysate Fortigel ${ }^{\circledR}$ from Gelita applied in our dog study. The aromatic- and NH-region (shown here) as well as the aliphatic region (not shown) were used to determine the diffusion constant. F2 displays the chemical shift of the collagen protons (in ppm). F1 is the frequency axis which provides information about the diffusion constant of the collagen-fragments. 

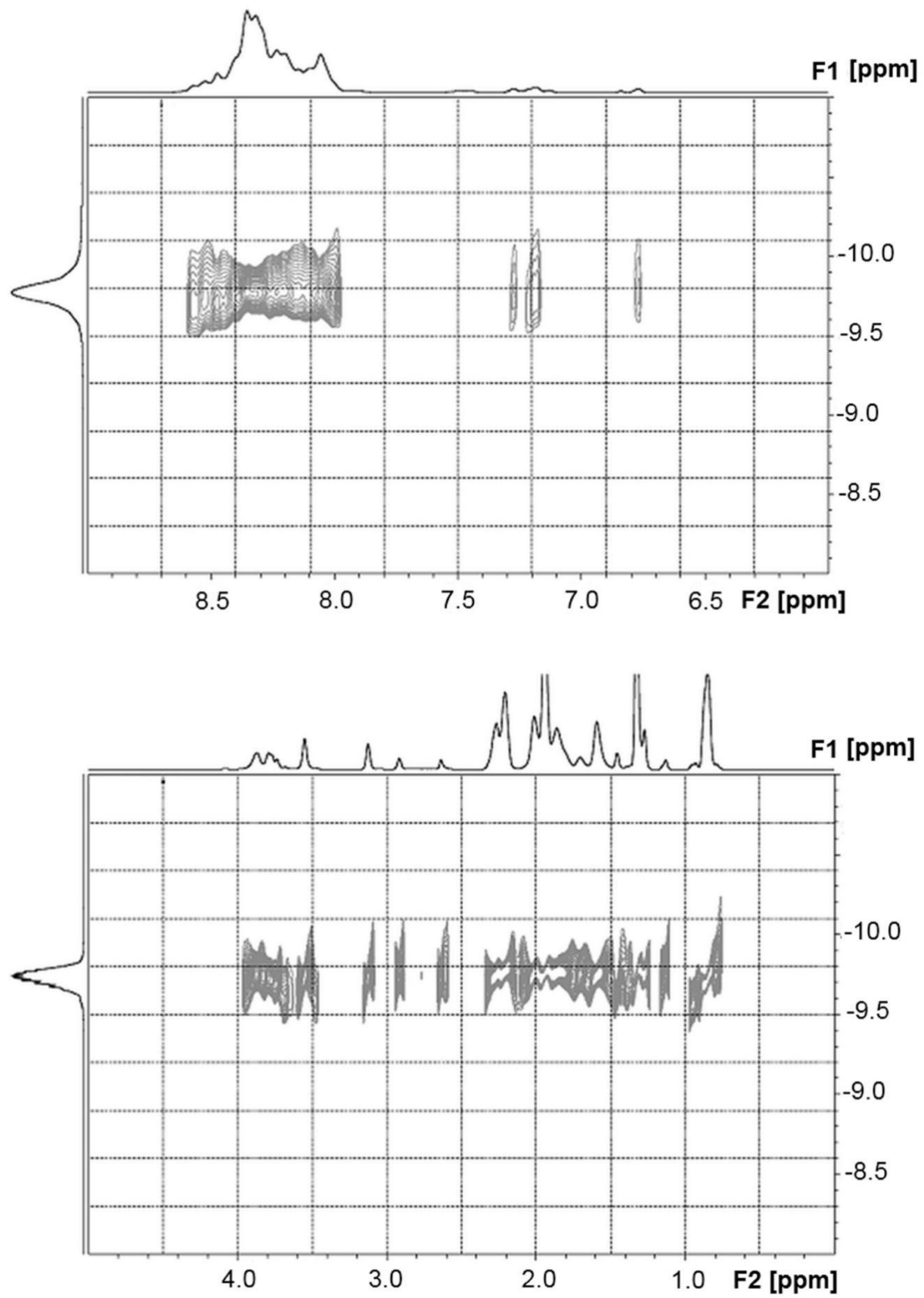

Figure 6. Parts of a two-dimensional NOESY spectrum of the used collagen-hydrolysate, Fortigel@. F1 and F2 are the frequency axis which display the chemical shifts of the collagen protons (in ppm). 


\subsection{Molecular Modeling.}

We utilized the available PDB structural data for MMP-3 (2JT6.pdb) and ADAMTS-5 (2RJQ.pdb) as the starting geometries for molecular modeling tasks. Although the experimental geometries of these proteins exhibit ligands in their binding sites, we were also interested, how sialic acid and GlcNAc in standard and sulfated [49] forms can bind to MMP-3 and ADAMTS-5. Thus, we were equally interested in the prediction of all possible binding sites (BS) for these two proteins. Three BS were predicted using the SiteMap [50] program for MMP-3 whereas the number of predicted BS for ADAMTS-5 was three times higher. The number of predicted binding sites is in accord with the size/weight of these proteins (18.54 kDa for MMP-3 and 42.84 $\mathrm{kDa}$ for ADAMTS-5).

\section{A}
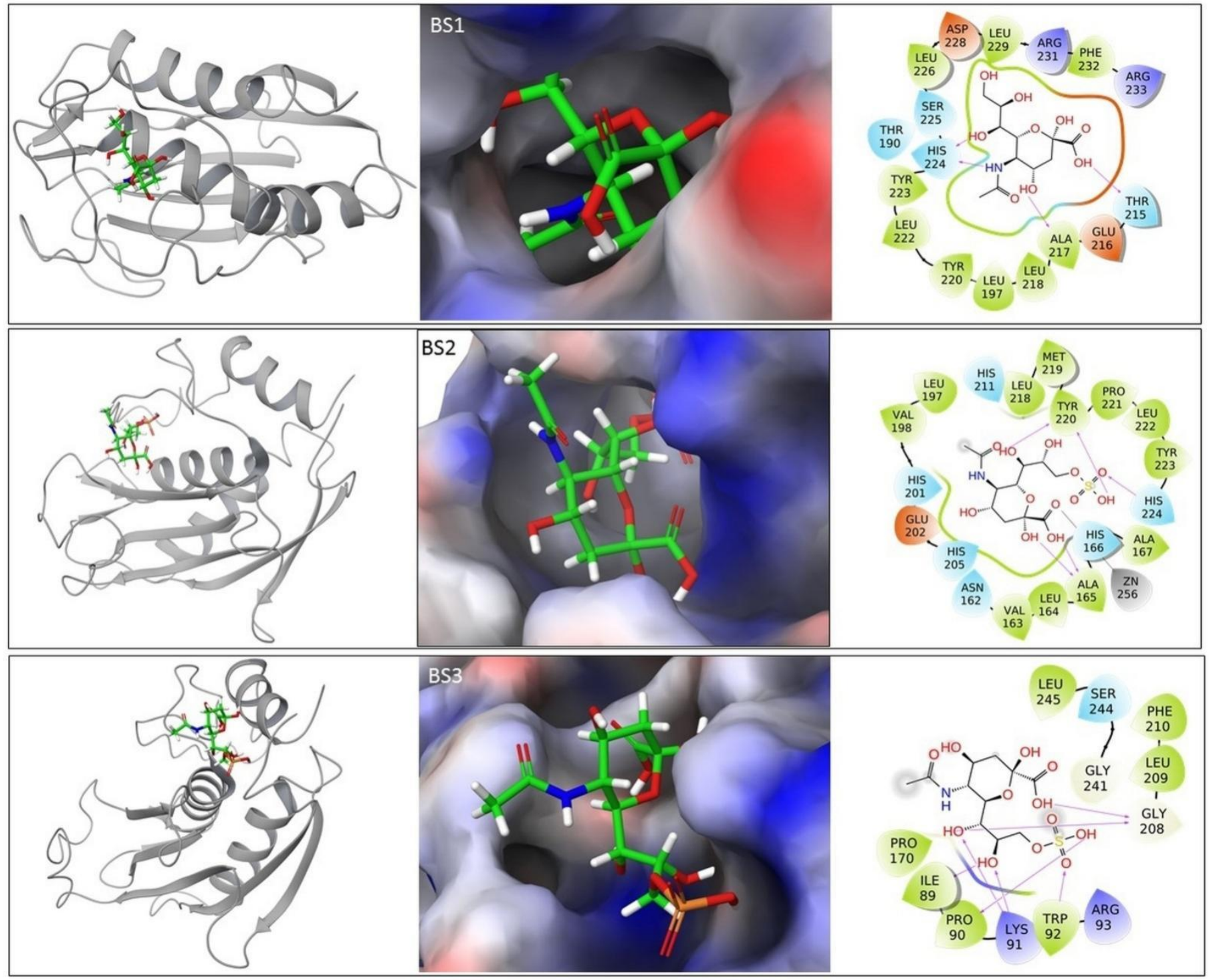
B

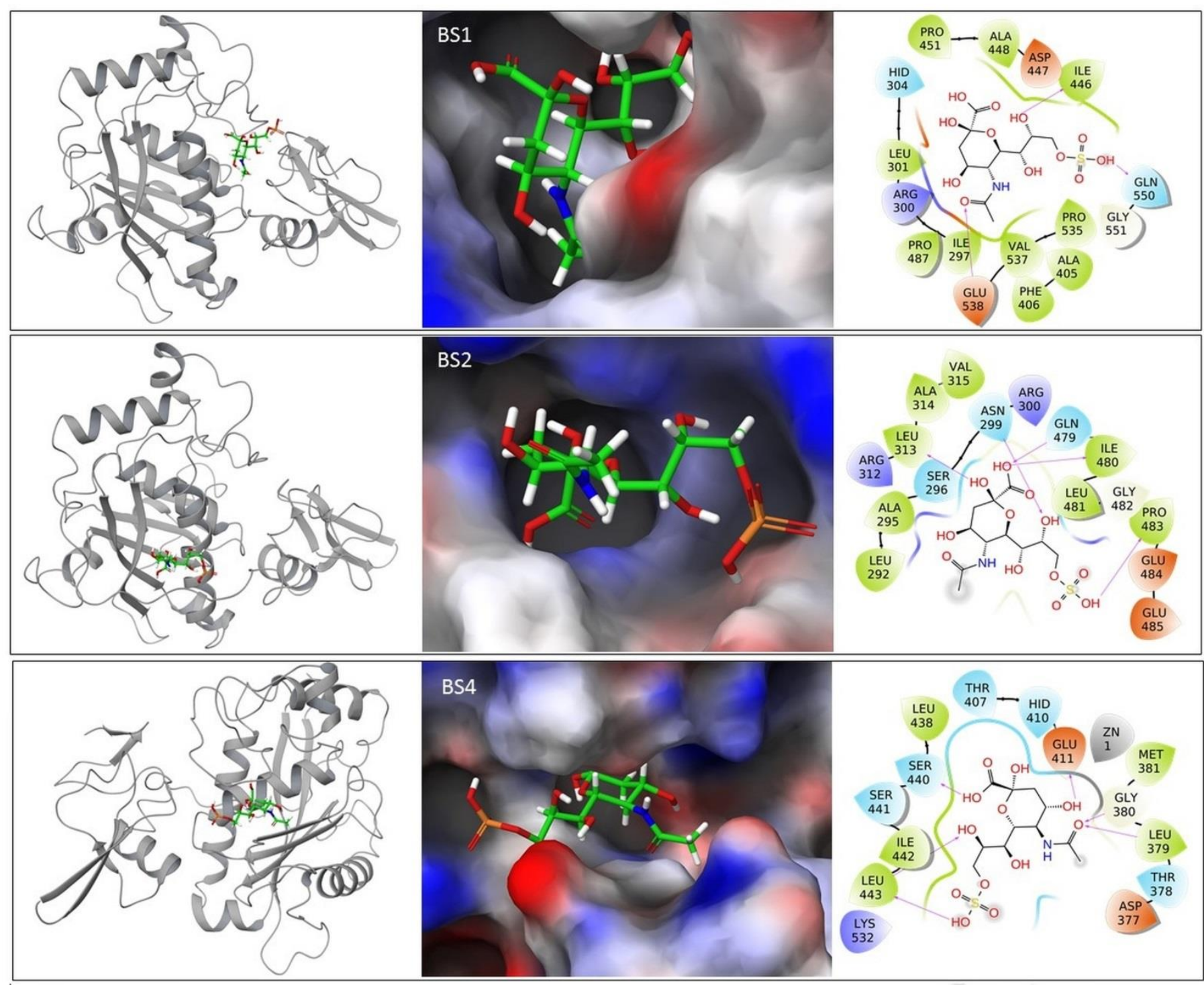

Figure 7. Glucosamines in binding sites of A) MMP-3 (2JT6.pdb) and B) ADAMTS-5 (2RJQ.pdb). The protein-ligand complexes are shown in ribbon representation in the left set of figures; the protein surfaces (colored according the electrostatic potential) zoomed into binding sites are in the middle set of figures whereas the details of the proteinligand interaction profiles are visualized in the right set of figures.

In the next step we calculated how N-Acetyl glucosamine (GlcNAc) and N-Acetyl neuraminic acid (Neu5Ac) (both in standard and sulfated forms) can bind into all predicted binding sites. We used the Glide [51] program to determine binding poses and the energetics of binding for the four carbohydrates. 
The Glide analysis for all binding sites and all carbohydrates resulted in more than one thousand proteinligand complexes. Table 2 presents the extracted lowest energy binding poses for MMP-3 and ADAMTS-5. Figure 7 shows the corresponding graphical outcome.

TABLE 2. Summary of the lowest docking scores for carbohydrate-protein complexes for all predicted binding sites. The lower value in comparision standard versus sulfated form of the carbohydrates is shown in italic; the lowest binding scores comparison the four carbohydrates in the binding sites (shown in table rows) are highlighted bold. The preferred binding sites for both proteins maked ${ }^{*}$ are visualized in Figure 7.

\begin{tabular}{|c|c|c|c|c|c|c|}
\hline \multirow[b]{2}{*}{ Protein } & \multirow[b]{2}{*}{$\begin{array}{l}\text { PDB } \\
\text { code }\end{array}$} & \multirow[b]{2}{*}{$\begin{array}{c}\text { Bind- } \\
\text { ing } \\
\text { Site }\end{array}$} & \multicolumn{4}{|c|}{ Docking Score [kcal/mol] } \\
\hline & & & GIcNAc & $\begin{array}{c}\text { GlcNAc- } \\
\text { sulf }\end{array}$ & Neu5Ac & $\begin{array}{c}\text { Neu5Ac- } \\
\text { sulf }\end{array}$ \\
\hline \multirow{3}{*}{ MMP-3 } & \multirow{3}{*}{ 2JT6 } & $\mathrm{BS}^{*}$ & -8.16 & -8.23 & $-11.48^{*}$ & -8.76 \\
\hline & & $\mathrm{BS}^{*}$ & -9.20 & -9.12 & -8.68 & $-9.43^{*}$ \\
\hline & & $\mathrm{BS}^{*}$ & -5.24 & -6.27 & -6.88 & $-7.42^{*}$ \\
\hline \multirow{9}{*}{ ADAMTS-5 } & \multirow{9}{*}{$2 \mathrm{RJQ}$} & $\mathrm{BS}^{*}{ }^{*}$ & -6.28 & -6.96 & -7.61 & $-8.09^{*}$ \\
\hline & & $\mathrm{BS} 2^{*}$ & -7.05 & -7.94 & -9.00 & $-9.22^{*}$ \\
\hline & & BS3 & -5.94 & -5.45 & -5.87 & -5.78 \\
\hline & & $\mathrm{BS}^{*}$ & -7.25 & -9.16 & -8.61 & $-9.35^{*}$ \\
\hline & & BS5 & -6.99 & -5.73 & -8.61 & -7.73 \\
\hline & & BS6 & & & -4.47 & -4.89 \\
\hline & & BS7 & -7.07 & -7.31 & -8.35 & -8.88 \\
\hline & & BS8 & -6.07 & -5.85 & -8.86 & -8.19 \\
\hline & & BS9 & -6.68 & -6.81 & -8.82 & -7.93 \\
\hline
\end{tabular}

The ribbon presentation for MMP-3 together with ligand-protein interaction analysis is illustrated in Figure 7A. Equivalent figures for ADAMTS-5 are presented on Figure-7B.

It is interesting to note the Neu5Ac is predicted to bind preferably (but ADAMTS-5 in BS3) over GlcNAc. The sulfated forms of glucosamines bind in the majority of cases better than the unsulfated molecules. Exception is Neu5Ac in BS1 of MMP-3. This is a special case (illustrated of Figure 7A-BS1) because this binding site is below the protein loop and the carbohydrate molecules thus interacts with the amino acids of the loop.

The next modeling step dealt with explicit modeling of the proteins with collagen fragments. We used the HEX [52] program here to generate (based on shape and electrostatics complementarity) around 100 protein-collagen complexes for both proteins. The lowest-energy forms from the HEX modeling were used as the starting structures for molecular dynamics (MD) simulations. 
MD simulation were performed in a water environment in order to evaluate the stability of the proteincollagen complexes. Figure 8 presents part of the results from $50 \mathrm{~ns}$ simulation. The structure at the simulation start plus 10 time-dependent structures extracted from the saved simulation trajectory at $5 \mathrm{~ns}$ intervals were superposed and are shown on the Figure 8 in order to present the time-evolved conformational changes. The "simulation quality analysis" of Maestro/Desmond (Figure 8A and 8C) indicated that the standard deviation of all analyzed parameters like total energy, potential energy or volume is below $0.01 \%$ of the average values variables that were a result of the MD simulations.

A

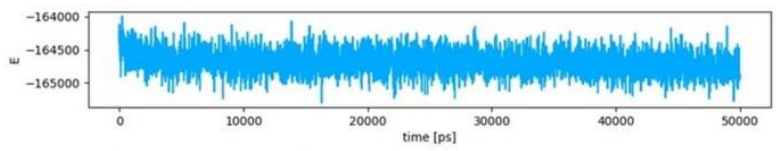

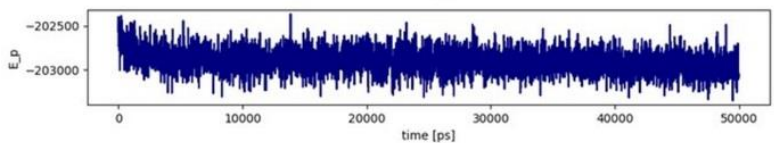

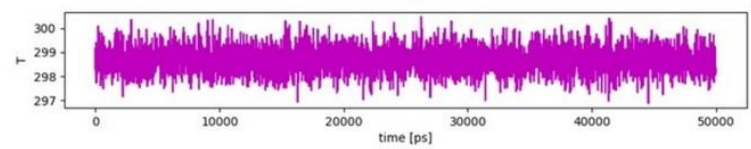

$\underbrace{0-\underbrace{}_{100}}_{0}$

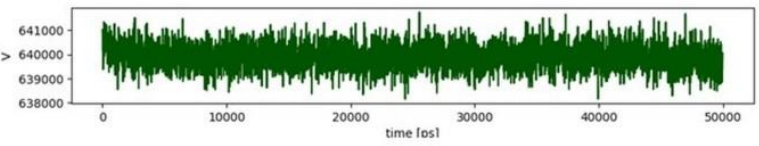

C
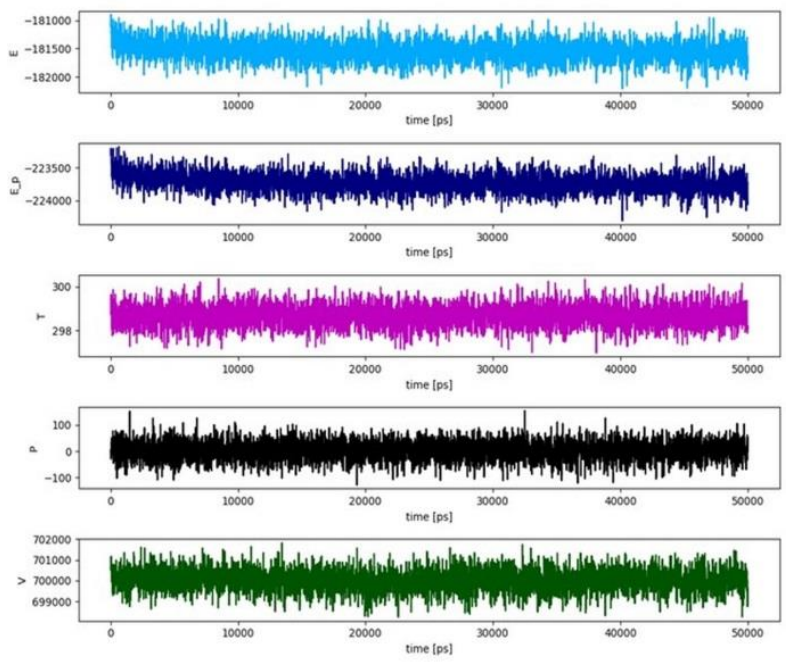

B

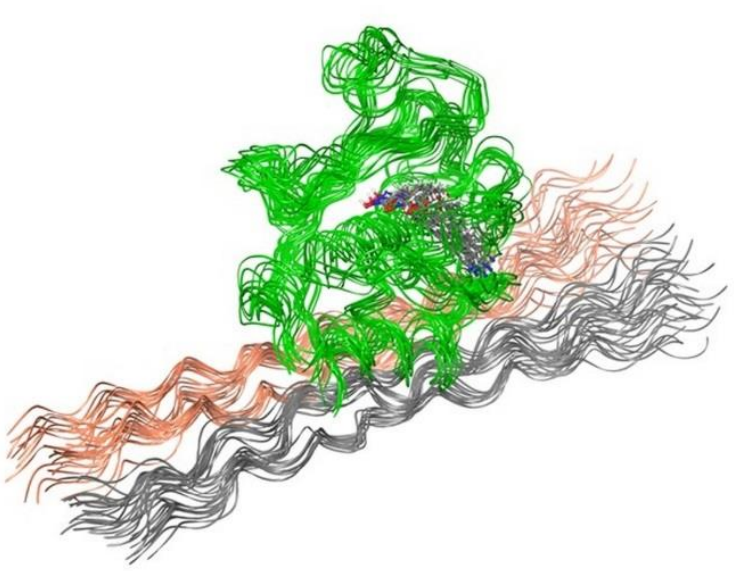

D

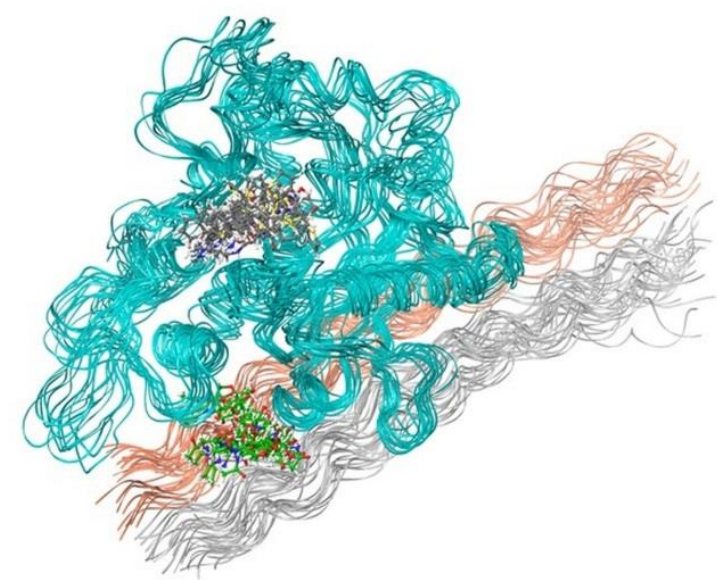

Figure 8. Molecular dynamics simulation results of solvated MMP-3/collagen (starting structure based on 2JT6.pdb + 1EI8.pdb) and ADAMTS-5/collagen (starting structure based on 2RJQ.pdb + 1EI8.pdb) 
supramolecular complexes. A,C - Simulation quality analysis of $50 \mathrm{~ns}$ DESMOND simulation as visualized in Maestro. Color coding: blue - total energy of the simulated system; dark blue - potential energy; violet - temperature; black - pressure; dark green - volume. B, D - Superpositions of protein-collagen structures resulted from the MD simulations. Only selected structures (shown as ribbons) of the supramolecular complexes saved in $5 \mathrm{~ns}$ time intervals are visualized. The ligands of the proteins are shown in ball\&stick models (grey carbons) whereas the GlcNAc present in ADAMTS-5 is shown with green carbons. The ribbons for A, B, C chains of the collagen fragments are visualized grey; the ribbons of D,E,F chains are shown in orange. The water molecules/ions present in the solvation box are not visualized

PLIP analysis and the consequent Access processing of the MD trajectory geometries allowed a comparison of overall hydrogen bonding versus hydrophobic interactions for both MMP-3/collagen and ADAMTS-5 collagen. Similar data was obtained with both systems with hydrogen bonding predominating with an incidence of $68 \%$ for ADAMTS-5 and $66 \%$ for MMP-3 complexes. In comparison, hydrophobic interaction stabilization accounted for $34 \%$ in the case of MMP-3 and 32\% in the case of ADAMTS-5.

The carbohydrate entity present on the protein surface in the case of ADAMTS-5 interacts with the collagen structure as shown in Figure 8B and 8D. Accordingly, the sulfate groups present at the glycan chains of proteoglycans can mediate interactions with collagen-triple helix structures of collagen present in cartilage.

\section{Discussion}

Our combined clinical, cell biological, biochemical, biophysical and molecular modeling approach on canine and equine patients is a feasible strategy to answer a number of questions related to collagen-hydrolysates, sulfated glycans and lipids as chondroprotective food supplements. Beyond this it is of highest importance that specific collagen-fragments were able to interact with various receptors (e.g. angiotensin receptors and sialic acid containing carbohydrate chains) in a specific way.

Although the collagen hydrosylate under study in contrary to the sulfated glucosamine and the lipids (utilized as components of the special dog food) did not originate from marine organisms we can assume comparably positive effects on cartilage health if collagen-hydrolysate of marine organisms (fish or jellyfish e.g. $[4,8,10])$ would be administered.

Articular cartilage destruction is mediated by the loss of collagen type II and proteoglycans and this loss is a characteristic feature of osteoarthritis symptoms. Our results show that it is possible to correlate the influence of collagen-hydrolysates on cartilage tissues [30-32] through specific biochemical pathways and cell-biological processes [1,4,5]. We found that collagen-hydrolysates were able to alter the levels of MMP-3 (as well as Matrix Metallo-Proteinase-1 and -13; Figure 4) without changing the level of TIMP-1 (Tissue Inhibitors of MetalloProteinases-1; data not shown). In addition, we recognized the involvement of collagen-hydrolysates and sulfated glucosamine in several key biochemical processes which are directly correlated with cartilage health. As noted, collagen-hydrolysates contain mixtures of collagen-fragments of various length.

We show here that modelling is a useful tool in evaluating specific interactions in protein binding sites and between these proteins and collagen fragments. No doubt further investigation on the impact of these interactions on protein activity in relation to targeting diseases will be crucial in further evaluating their effect on the extracellular matrix and therapeutic value. 


\section{Materials and Methods.}

Sulfated GlcNAc in form of tablets, collagen-hydrolysate from Gelita dry powder, Hills JD high quality dogfood were used to target dog's osteoarthritis.

\subsection{Cell Biological Test.}

The methods used are the same as described in our former publications Raabe et al. [8] and Zhang et al. [24]. We focused on the impact of collagen-hydrolysates on the differentiation of chondrocytes as well as on the role of sialic acids as contact structures of the cell surface as differentiation markers.

\subsection{Blood Parameter Determination.}

Blood samples were obtained from all dogs under study in order to clarify any existing medical condition and to ensure that there was no acute Lyme disease. The blood samples were taken after stasis and disinfection with $70 \%$ alcohol on the anterior vein cephalic with a $7.5 \mathrm{ml} \mathrm{S}$-Monovette (Sarstedt) and attached cannula (Sarstedt). 16 IU served as anticoagulant Heparin. The blood was centrifuged at $3000 \mathrm{rpm}$ for 10 minutes after collection. A large blood count (hematology), an organ profile and an IgG / IgM borreliosis antibody titer were created for each dog in the Synlab Augsburg laboratory. Blood was drawn as part of the treatment. A portion of this blood was portioned for the determination of the cartilage markers and shipped to Gießen to examine special osteoarthritis markers. The plasma for the osteoarthritis markers was placed in an Eppendorf cone with an Eppendorf pipette. The samples obtained were temporarily stored at $-20^{\circ} \mathrm{C}$ for a maximum of 10 days after labeling and then sent by post using dry ice and stored at $-70^{\circ} \mathrm{C}$ until evaluation. Cartilage markers were analyzed as published [53].

\subsection{Statistical Analysis.}

The data obtained is summarized using Microsoft Excel (Office 2000 package). The statistical data is also prepared using Excel. Missing data are marked with * in our study protocols, so this entry is treated by BMDP as a missing value. The data was evaluated on the computers in the local computer network (LAN) of the Biomathematics and Data Processing working group of the Veterinary Medicine Department of the Justus Liebig University in Gießen. The statistical evaluations were carried out using the statistical program package BMDP / Dynamic, Release 8.1 (Dixon, 1993). Arithmetic mean values (8x), standard deviations (s), minima $\left(\mathrm{x}_{\min }\right)$, maxima $\left(\mathrm{x}_{\max }\right)$ and sample sizes $(\mathrm{n})$ were calculated and presented in tabular form to describe the data for quantitative, approximately normally distributed characteristics. The qualitative characteristics were counted according to groups and presented in the form of frequency tables. A two-factor analysis of variance with repeated measurements of time with the BMDP2V program was carried out in groups $1+2$ for statistically checking the influence of group and time on significance. If values were missing, this was done using the BMDP5V (so-called "forest test"). With regard to the quantitative characteristics, the t-test and otherwise the Wilcoxon-Mann-Whitney test (BMDP3D) were used in the group comparison of these two groups with normal distribution. For the semi-quantitative characteristics, the exact Wilcoxon-Mann-Whitney test using the "StatXact" program was used when comparing the two groups. For the comparison of qualitative charac- 
teristics, frequency tables were generated for all 3 groups with the BMDP4F program. The qualitative characteristics were again checked for significant correlations for groups $1+2$ with the exact test from Fisher for each point of time. The Fisher-Freeman-Halton test was used for more than 2 versions. The "StatXact" program was used here (Cytel, 2010). The significance level $\alpha=0.05$ was used as the basis for evaluating the statistical significance, i.e. Results with $\mathrm{p} \leq 0.05$ were stated to be statistically significant. In addition, the exact p-value was given if possible. Group 3 was not included in the significance calculation as a trailing control group.

\subsection{Nuclear Magnetic Resonance (NMR) Spectroscopy.}

Proton NMR was applied to analyze the Fortigel collagen-hydrolysate in terms of its fragment size distribution (DOSY) and possible identification of certain amino acid types (TOCSY / NOESY). In the NMR tubes collagen-hydrolysates were dissolved at an amount of $3 \mathrm{mg}$ in $0.5 \mathrm{ml}$ water $\left(90 \% \mathrm{H}_{2} \mathrm{O} / 10 \% \mathrm{D}_{2} \mathrm{O}\right)$. The NMR experiments were performed on a $600 \mathrm{MHz}$ Bruker Avance III spectrometer at 298K. 2D-TOCSY experiments (DIPSI-2; mixing time $80 \mathrm{~ms}$ ) and 2D-NOESY (mixing times 200 or $400 \mathrm{~ms}$ ) were recorded with 512 (F1) x 1024 (F2) complex data points and a spectral widths of $7212 \mathrm{~Hz}$ (12 ppm). Water suppression was performed using excitation sculpting and per increment 16 scans were accumulated with an inter-scan recovery delay of 1.5 s. For processing we used zero-filling to 1024 (F1) x 2048 (F2) data points prior to Fourier transformation, followed by baseline correction in both dimensions. Spectra were calibrated on internal water.

\subsection{Molecular Modeling.}

The structures of glucosamines, i.e. N-Acetyl Glucosamine (GlcNAc) and N-Acetyl Neuraminic acid (Neu5Ac; also sialic acid) were downloaded from PUBCHEM (https://pubchem.ncbi.nlm.nih.gov/) in SDF format and imported into Maestro V. 12.3.013 [54] project table. Both molecules were then sulfated using the Maestro molecular builder option. Their molecular structures are presented in Scheme 1. Advanced conformational search for carbohydrate side chain orientations were then carried out maintaining their ${ }^{4} \mathrm{C}_{1}$ forms for GlcNAc and GlcNAc-sulf and ${ }^{1} \mathrm{C}_{4}$ for Neu5Ac and Neu5Ac-sulf ring conformations. The geometries of five selected low-energy conformations of the four carbohydrates were ab initio (DFT B3LYP 6-31G**) optimized (releasing all geometric parameters) with the Gaussian [55] program (G09 version) and were then used as input ligands for molecular docking into matrix proteins like MMP-3 and aggrecanases (i.e. ADAMTS-5), present in the dog and horses organisms. 


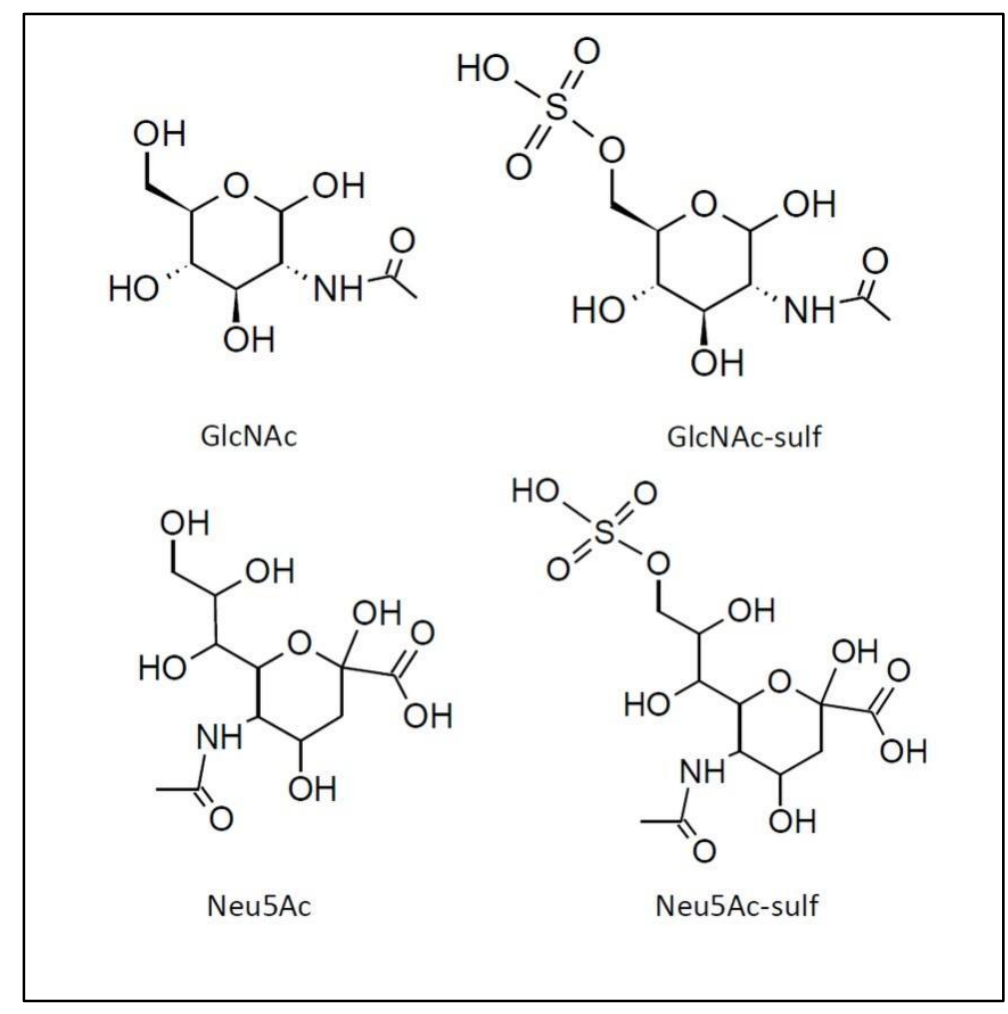

Scheme 1. Molecular structures of GlcNAc and Neu5Ac in standard and sulfated forms.

The atomic coordinates of MMP-3 (2JT6.pdb [14]), ADAMTS-5 (2RJQ.pdb [13]) and a collagen fragment (1EI8.pdb [56]) structures were downloaded from the protein database and imported into Maestro. All protein geometries were processed (adding missing atoms, fixing bond orders, assign partial charges) by "Protein Preparation Wizard" of the Maestro program [54].

The possible binding sites of MMP-3 and ADAMTS-5 were evaluated using the SiteMap program [50,57] of Schrodinger Inc. The G09-optimized conformations of four ligands (Scheme 1) were docked into all SiteMap predicted binding sites of MMP-3 and ADAMTS-5 using the GLIDE program [51,58-60]. While flexible docking was considered for all side chains of the carbohydrates, the pyranose rings were fixed in ${ }^{4} \mathbf{C}_{1}$ for ${ }^{1} \mathbf{C}_{4}$ forms as stated above. All protein-ligand complexes resulting from GLIDE flexible were optimized (OPLS-2005 force field) and were tableted/ordered according their GLIDE docking scores.

The HEX program [52] was used to generate and preoptimize the geometries of the MMP-3/collagen and ADAMTS-5/collagen supramolecular complexes. From around 100 generated complexes the lowest-energy ones were selected for further molecular dynamics (MD) studies.

These HEX-output files of MMP-3/collagen and ADAMTS-5/collagen complexes were imported into Maestro and were processed by standard methods (as indicated above by "Protein Preparation Wizard") to get the structures prepared for MD runs using the Desmond [54,61] program The OPLS-2005 force field (the upto-date version of the OPLS force field family $[62,63]$ ) was used to carry out the simulation studies. The protein-collagen complexes were solvated at first in Maestro (SPC water model [64]; the water molecules were added within $1 \mathrm{~nm}$ buffer around the proteins) and the resulting structures were then minimized and equilibrated for $5 \mathrm{~ns}$. The final structures after equilibration were submitted for $50 \mathrm{~ns}$ NPT (pressure at 1.01325 
bar) molecular dynamics (MD) simulations with the Desmond program at $300 \mathrm{~K}$. Molecular geometries resulting from simulations were saved at $10 \mathrm{ps}$ intervals and were used for further analysis. These geometries (5000 altogether) from each simulation were exported into $\mathrm{pdb}$ format and were used for analysis of interaction profiles using the PLIP program [65]. The xml files resulted from the PLIP calculation were imported into Microsoft Access for analysis and data mining.

Schrodinger's Maestro [54] was used for visualization of molecular structures, their complexes and protein-carbohydrate ligand interaction profiles.

\section{Conclusions.}

Both biophysical and computational methods were found to be useful tools in providing valuable information on the relationship between therapeutic value and mechanism. Such approaches will be key prior to their or other similar compounds being adopted further as potential medical therapies.

The likelihood for the tested nutraceuticals to be valuable chondroprotective substances which can aid in the prevention of early osteoarthritis symptoms is clearly indicated in our clinical study. We propose that the most promising application of these nutraceuticals would be a combination of collagen-hydrolysate and sulfated glucosamine as a supplement to food source rich in lipids and vitamins. The special role of sulfate groups, sialic acids and other carbohydrate contact structures in the context of binding affinities in respect to certain protein- and peptide- structures, especially, collagen-fragments is discussed in the literature and will be further examined in a follow-up study.

Acknowledgements. We thank Prof. Dr. Ralf Boelens, Dr. Hans Wienk (University Utrecht (NL)), Dr. Steffen Oesser (CRI Kiel), Prof. Dr. Martin Kramer, Prof. Dr. Lutz-F. Litzke, Dr. Klaus Failing, Dr. Uschi Siebler (FB 10 University Gießen) and Prof. Dr. Eckhard Wolf (Faculty of Veterinary Medicine, Gene Center and Department of Biochemistry, Ludwig-Maximilians University, Munich, Germany) for scientific support and Philipp Siebert (RI-B-NT) for technical assistance. Dr. Werner Jahn from the horse clinic in Bargteheide (Schleswig-Holstein, Germany) is thanked for valuable discussions concerning arthritic therapies on horses as well as Mrs. Shaida von Berenberg-Gossler (Hamburg, Germany) for her important hints about the behavior of dressage horses as well as Dr. Götz Dreismann (Practice for small animals and horses, Tornesch, SchleswigHolstein, Germany) for fruitful discussions about sulfated glucosamines. Prof. Dr. Ivano Bertini who unfortunately passed away in 2012 was over years at many scientific meetings an inspiring discussion partner concerning his pioneering NMR work in the field of matrix metalloproteinases and paramagnetic molecules. We also thank the company Gelita for collagen-hydrolysates.

Funding. Elements of the project are financed by the European Commission's Framework Program 7 (Bio-NMR; project number 261863). Doctoral Foundation of Liaocheng University (No. 318051738 and No. 318051827), National Natural Science Foundation of China (No. 82001286), Natural Science Foundation of Shandong province (No.ZR2020QH112), and Taishan Scholar Research Foundation. This publication is the result of the project implementation: Open Scientific Community for Modern Interdisciplinary Research in Medicine (OPENMED), ITMS2014+: 313011V455 supported by the Operational Programme Integrated Infrastructure, funded by the ERDF.

Conflicts of interest. The authors declare that they have no conflict of interest. 
Ethical statement. All animal procedures were performed after the permission of each patient holder as a clinical trial study and after its indication at the corresponding administration offices in charge at the JustusLiebig-University Gießen (Hessen, Germany).

\section{References}

1. Siebert, H.C.; Burg-Roderfeld, M.; Eckert, T.; Stötzel, S.; Kirch, U.; Diercks, T.; Humphries, M.J.; Frank, M.; Wechselberger, R.; Tajkhorshid, E., et al. Interaction of the alpha2A domain of integrin with small collagen fragments. Protein Cell 2010, 1, 393-405, doi:10.1007/s13238-010-0038-6.

2. Krylov, V.B.; Grachev, A.A.; Ustyuzhanina, N.E.; Ushakova, N.A.; Preobrazhenskaya, M.E.; Kozlova, N.I.; Portsel, M.N.; Konovalova, I.N.; Novikov, V.Y.; Siebert, H.C., et al. Preliminary structural characterization, antiinflammatory and anticoagulant activities of chondroitin sulfates from marine fish cartilage. Russian Chem Bull 2011, 60, 746-753, doi:10.1007/s11172-011-0115-x.

3. Burg-Roderfeld, M.; Eckert, T.; Siebert, H.C. Bioaktive Kollagenfragmente. Neue struktur-biologische Studien an Kollagen-Integrin-Komplexen belegen Justus Liebigs wegweisende Ideen. . Spiegel der Forschung 2011, 28, 36-43.

4. Stötzel, S.; Schurink, M.; Wienk, H.; Siebler, U.; Burg-Roderfeld, M.; Eckert, T.; Kulik, B.; Wechselberger, R.; Sewing, J.; Steinmeyer, J., et al. Molecular organization of various collagen fragments as revealed by atomic force microscopy and diffusion-ordered NMR spectroscopy. ChemPhysChem 2012, 13, 3117-3125, doi:10.1002/cphc.201200284.

5. Schadow, S.; Simons, V.S.; Lochnit, G.; Kordelle, J.; Gazova, Z.; Siebert, H.C.; Steinmeyer, J. Metabolic Response of Human Osteoarthritic Cartilage to Biochemically Characterized Collagen Hydrolysates. Int J Mol Sci 2017, 18, 207, doi:10.3390/ijms18010207.

6. Kosinska, M.K.; Ludwig, T.E.; Liebisch, G.; Zhang, R.; Siebert, H.C.; Wilhelm, J.; Kaesser, U.; Dettmeyer, R.B.; Klein, H.; Ishaque, B., et al. Articular Joint Lubricants during Osteoarthritis and Rheumatoid Arthritis Display Altered Levels and Molecular Species. PLoS One 2015, 10, e0125192, doi:10.1371/journal.pone.0125192.

7. Schadow, S.; Siebert, H.C.; Lochnit, G.; Kordelle, J.; Rickert, M.; Steinmeyer, J. Collagen metabolism of human osteoarthritic articular cartilage as modulated by bovine collagen hydrolysates. PLoS One 2013, 8, e53955, doi:10.1371/journal.pone.0053955.

8. Raabe, O.; Reich, C.; Wenisch, S.; Hild, A.; Burg-Roderfeld, M.; Siebert, H.C.; Arnhold, S. Hydrolyzed fish collagen induced chondrogenic differentiation of equine adipose tissue-derived stromal cells. Histochem Cell Biol 2010, 134, 545-554, doi:10.1007/s00418-010-0760-4.

9. Oke, S.; Aghazadeh-Habashi, A.; Weese, J.S.; Jamali, F. Evaluation of glucosamine levels in commercial equine oral supplements for joints. Equine Vet J 2006, 38, 93-95, doi:10.2746/042516406775374306.

10. Eckert, T.; Stötzel, S.; Burg-Roderfeld, M.; Sewing, J.; Lütteke, T.; E. Nifantiev, N.; F. G. Vliegenthart, J.; Siebert, H.-C. In silico Study on Sulfated and Non-Sulfated Carbohydrate Chains from Proteoglycans in Cnidaria and Interaction with Collagen. Open J. Phys. Chem. 2012, 02, 123-133, doi:10.4236/ojpc.2012.22017.

11. Benavente, M.; Arias, S.; Moreno, L.; Martínez, J. Production of Glucosamine Hydrochloride from Crustacean Shell. J Pharm Pharmacol 2015, 3, 20-26, doi:10.17265/2328-2150/2015.01.003.

12. Bertini, I.; Calderone, V.; Cosenza, M.; Fragai, M.; Lee, Y.M.; Luchinat, C.; Mangani, S.; Terni, B.; Turano, P. Conformational variability of matrix metalloproteinases: beyond a single $3 \mathrm{D}$ structure. Proceedings of the National Academy of Sciences of the United States of America 2005, 102, 5334-5339, doi:10.1073/pnas.0407106102. 
13. Mosyak, L.; Georgiadis, K.; Shane, T.; Svenson, K.; Hebert, T.; McDonagh, T.; Mackie, S.; Olland, S.; Lin, L.; Zhong, X., et al. Crystal structures of the two major aggrecan degrading enzymes, ADAMTS4 and ADAMTS5. Protein science : a publication of the Protein Society 2008, 17, 16-21, doi:10.1110/ps.073287008.

14. Alcaraz, L.A.; Banci, L.; Bertini, I.; Cantini, F.; Donaire, A.; Gonnelli, L. Matrix metalloproteinase-inhibitor interaction: the solution structure of the catalytic domain of human matrix metalloproteinase-3 with different inhibitors. Journal of biological inorganic chemistry : JBIC : a publication of the Society of Biological Inorganic Chemistry 2007, 12, 1197-1206, doi:10.1007/s00775-007-0288-9.

15. Iyer, S.; Wei, S.; Brew, K.; Acharya, K.R. Crystal structure of the catalytic domain of matrix metalloproteinase-1 in complex with the inhibitory domain of tissue inhibitor of metalloproteinase-1. The Journal of biological chemistry 2007, 282, 364-371, doi:10.1074/jbc.M607625200.

16. Sadowski, T.; Steinmeyer, J. Effects of tetracyclines on the production of matrix metalloproteinases and plasminogen activators as well as of their natural inhibitors, tissue inhibitor of metalloproteinases-1 and plasminogen activator inhibitor-1. Inflammation research : official journal of the European Histamine Research Society ... [et al.] 2001, 50, 175-182, doi:10.1007/s000110050742.

17. Masuyer, G.; Schwager, S.L.; Sturrock, E.D.; Isaac, R.E.; Acharya, K.R. Molecular recognition and regulation of human angiotensin-I converting enzyme (ACE) activity by natural inhibitory peptides. Sci Rep 2012, 2, 717, doi:10.1038/srep00717.

18. Kawakami, Y.; Matsuo, K.; Murata, M.; Yudoh, K.; Nakamura, H.; Shimizu, H.; Beppu, M.; Inaba, Y.; Saito, T.; Kato, T., et al. Expression of Angiotensin II Receptor-1 in Human Articular Chondrocytes. Arthritis 2012, 2012, 648537, doi:10.1155/2012/648537.

19. Nakamura, F.; Tsukamoto, I.; Inoue, S.; Hashimoto, K.; Akagi, M. Cyclic compressive loading activates angiotensin II type 1 receptor in articular chondrocytes and stimulates hypertrophic differentiation through a G-protein-dependent pathway. FEBS Open Bio 2018, 8, 962-973, doi:10.1002/2211-5463.12438.

20. Kouguchi, T.; Ohmori, T.; Shimizu, M.; Takahata, Y.; Maeyama, Y.; Suzuki, T.; Morimatsu, F.; Tanabe, S. Effects of a chicken collagen hydrolysate on the circulation system in subjects with mild hypertension or high-normal blood pressure. Biosci Biotechnol Biochem 2013, 77, 691-696, doi:10.1271/bbb.120718.

21. Bhunia, A.; Vivekanandan, S.; Eckert, T.; Burg-Roderfeld, M.; Wechselberger, R.; Romanuka, J.; Bächle, D.; Kornilov, A.V.; von der Lieth, C.-W.; Jiménez-Barbero, J.s., et al. Why Structurally Different Cyclic Peptides Can Be Glycomimetics of the HNK-1 Carbohydrate Antigen []. J Am Chem Soc 2010, 132, 3636-3636, doi:10.1021/ja100344v.

22. Toegel, S.; Pabst, M.; Wu, S.Q.; Grass, J.; Goldring, M.B.; Chiari, C.; Kolb, A.; Altmann, F.; Viernstein, H.; Unger, F.M. Phenotype-related differential alpha-2,6- or alpha-2,3-sialylation of glycoprotein N-glycans in human chondrocytes. Osteoarthritis Cartilage 2010, 18, 240-248, doi:10.1016/j.joca.2009.09.004.

23. Schauer, R.; Kamerling, J.P. Exploration of the Sialic Acid World. Adv Carb Chem Biochem 2018, 75, 1-213, doi:10.1016/bs.accb.2018.09.001.

24. Zhang, R.; Loers, G.; Schachner, M.; Boelens, R.; Wienk, H.; Siebert, S.; Eckert, T.; Kraan, S.; Rojas-Macias, M.A.; Lütteke, T., et al. Molecular Basis of the Receptor Interactions of Polysialic Acid (polySia), polySia Mimetics, and Sulfated Polysaccharides. ChemMedChem 2016, 11, 990-1002, doi:10.1002/cmdc.201500609.

25. Siebert, H.-C.; Scheidig, A.; Eckert, T.; Wienk, H.; Boelens, R.; Mahvash, M.; Petridis, A.K.; Schauer, R. Interaction studies of sialic acids with model receptors contribute to nanomedical therapies. J. Neurol. Disord 2015, 3, 1-6. 
26. Siebert, H.-C.; Lu, S.-Y.; Wechselberger, R.; Born, K.; Eckert, T.; Liang, S.; Lieth, C.-W.v.d.; Jiménez-Barbero, J.; Schauer, R.; Vliegenthart, J.F.G., et al. A lectin from the Chinese bird-hunting spider binds sialic acids. Carbohydrate Research 2010, 344, 1515-1525, doi:10.1016/j.carres.2009.06.002.

27. Zhang, R.; Wu, L.; Eckert, T.; Burg-Roderfeld, M.; Rojas-Macias, M.A.; Lütteke, T.; Krylov, V.B.; Argunov, D.A.; Datta, A.; Markart, P., et al. Lysozyme's lectin-like characteristics facilitates its immune defense function. $Q R e v$ Biophys 2017, 50, e9, doi:10.1017/S0033583517000075.

28. Zhang, R.; Eckert, T.; Lütteke, T.; Hanstein, S.; Scheidig, A.; Bonvin, A.M.; Nifantiev, N.E.; Kozar, T.; Schauer, R.; Enani, M.A., et al. Structure-Function Relationships of Antimicrobial Peptides and Proteins with Respect to Contact Molecules on Pathogen Surfaces. Curr Top Med Chem 2016, 16, 89-98, doi:10.2174/1568026615666150703120753.

29. Kar, R.K.; Gazova, Z.; Bednarikova, Z.; Mroue, K.H.; Ghosh, A.; Zhang, R.; Ulicna, K.; Siebert, H.C.; Nifantiev, N.E.; Bhunia, A. Evidence for Inhibition of Lysozyme Amyloid Fibrillization by Peptide Fragments from Human Lysozyme: A Combined Spectroscopy, Microscopy, and Docking Study. Biomacromolecules 2016, 17, 1998-2009, doi:10.1021/acs.biomac.6b00165.

30. Oesser, S.; Adam, M.; Babel, W.; Seifert, J. Oral administration of (14)C labeled gelatin hydrolysate leads to an accumulation of radioactivity in cartilage of mice (C57/BL). J Nutr 1999, 129, 1891-1895, doi:10.1093/jn/129.10.1891.

31. Oesser, S.; Seifert, J. Stimulation of type II collagen biosynthesis and secretion in bovine chondrocytes cultured with degraded collagen. Cell Tissue Res 2003, 311, 393-399, doi:10.1007/s00441-003-0702-8.

32. Schunck, M.; Louton, H.; Oesser, S. The Effectiveness of Specific Collagen Peptides on Osteoarthritis in DogsImpact on Metabolic Processes in Canine Chondrocytes. Open J. Animal Sci. 2017, 07, 254-266, doi:10.4236/ojas.2017.73020.

33. Dobenecker, B.; Reese, S.; Jahn, W.; Schunck, M.; Hugenberg, J.; Louton, H.; Oesser, S. Specific bioactive collagen peptides (PETAGILE $((\mathrm{R}))$ ) as supplement for horses with osteoarthritis: A two-centred study. J Anim Physiol Anim Nutr (Berl) 2018, 102 Suppl 1, 16-23, doi:10.1111/jpn.12863.

34. Simons, V.S.; Lochnit, G.; Wilhelm, J.; Ishaque, B.; Rickert, M.; Steinmeyer, J. Comparative Analysis of Peptide Composition and Bioactivity of Different Collagen Hydrolysate Batches on Human Osteoarthritic Synoviocytes. Sci Rep 2018, 8, 17733, doi:10.1038/s41598-018-36046-3.

35. Porfírio, E.; Fanaro, G.B. Collagen supplementation as a complementary therapy for the prevention and treatment of osteoporosis and osteoarthritis: a systematic review. Revista Brasileira de Geriatria e Gerontologia 2016, 19, 153-164, doi:10.1590/1809-9823.2016.14145.

36. Li, R.; Qiao, S.; Zhang, G. Analysis of angiotensin-converting enzyme 2 (ACE2) from different species sheds some light on cross-species receptor usage of a novel coronavirus 2019-nCoV. The Journal of infection 2020, 80, 469-496, doi:10.1016/j.jinf.2020.02.013.

37. Saponaro, F.; Rutigliano, G.; Sestito, S.; Bandini, L.; Storti, B.; Bizzarri, R.; Zucchi, R. ACE2 in the Era of SARSCoV-2: Controversies and Novel Perspectives. Frontiers in Molecular Biosciences 2020, 7, doi:10.3389/fmolb.2020.588618.

38. Lam, S.D.; Bordin, N.; Waman, V.P.; Scholes, H.M.; Ashford, P.; Sen, N.; van Dorp, L.; Rauer, C.; Dawson, N.L.; Pang, C.S.M., et al. SARS-CoV-2 spike protein predicted to form complexes with host receptor protein orthologues from a broad range of mammals. Sci Rep 2020, 10, 16471, doi:10.1038/s41598-020-71936-5.

39. Zhang, N.; Liu, C.; Zhang, R.; Jin, L.; Yin, X.; Zheng, X.; Siebert, H.C.; Li, Y.; Wang, Z.; Loers, G., et al. Amelioration of clinical course and demyelination in the cuprizone mouse model in relation to ketogenic diet. Food Funct 2020, 11, 5647-5663, doi:10.1039/c9fo02944c. 
40. Zhang, N.; Liu, C.; Jin, L.; Zhang, R.; Siebert, H.C.; Wang, Z.; Prakash, S.; Yin, X.; Li, J.; Hou, D., et al. Influence of Long-Chain/Medium-Chain Triglycerides and Whey Protein/Tween 80 Ratio on the Stability of Phosphatidylserine Emulsions (O/W). ACS Omega 2020, 5, 7792-7801, doi:10.1021/acsomega.9b03702.

41. Woods, R.J. Predicting the Structures of Glycans, Glycoproteins, and Their Complexes. Chemical reviews 2018, 118, 8005-8024, doi:10.1021/acs.chemrev.8b00032.

42. Nivedha, A.K.; Makeneni, S.; Foley, B.L.; Tessier, M.B.; Woods, R.J. Importance of ligand conformational energies in carbohydrate docking: Sorting the wheat from the chaff. J Comput Chem 2014, 35, 526-539, doi:10.1002/jcc.23517.

43. Tsvetkov, Y.E.; Burg-Roderfeld, M.; Loers, G.; Arda, A.; Sukhova, E.V.; Khatuntseva, E.A.; Grachev, A.A.; Chizhov, A.O.; Siebert, H.C.; Schachner, M., et al. Synthesis and molecular recognition studies of the HNK-1 trisaccharide and related oligosaccharides. The specificity of monoclonal anti-HNK-1 antibodies as assessed by surface plasmon resonance and STD NMR. J Am Chem Soc 2012, 134, 426-435, doi:10.1021/ja2083015.

44. Belshaw, Z.; Asher, L.; Harvey, N.D.; Dean, R.S. Quality of life assessment in domestic dogs: An evidence-based rapid review. Veterinary journal 2015, 206, 203-212, doi:10.1016/j.tvjl.2015.07.016.

45. Hansen, B.D. Assessment of pain in dogs: veterinary clinical studies. ILAR J 2003, 44, 197-205, doi:10.1093/ilar.44.3.197.

46. Seales, E.C.; Jurado, G.A.; Singhal, A.; Bellis, S.L. Ras oncogene directs expression of a differentially sialylated, functionally altered beta1 integrin. Oncogene 2003, 22, 7137-7145, doi:10.1038/sj.onc.1206834.

47. Luo, Y.; Sinkeviciute, D.; He, Y.; Karsdal, M.; Henrotin, Y.; Mobasheri, A.; Onnerfjord, P.; Bay-Jensen, A. The minor collagens in articular cartilage. Protein Cell 2017, 8, 560-572, doi:10.1007/s13238-017-0377-7.

48. Kashiwagi, M.; Tortorella, M.; Nagase, H.; Brew, K. TIMP-3 is a potent inhibitor of aggrecanase 1 (ADAM-TS4) and aggrecanase 2 (ADAM-TS5). The Journal of biological chemistry 2001, 276, 12501-12504, doi:10.1074/jbc.C000848200.

49. Ertunc, N.; Sato, C.; Kitajima, K. Sialic acid sulfation is induced by the antibiotic treatment in mammalian cells. Biosci Biotechnol Biochem 2020, 84, 2311-2318, doi:10.1080/09168451.2020.1792763.

50. $\quad$ Schrodinger, L. SiteMap, version 3.9. SiteMap, version 3.92013.

51. Schrödinger, L. Glide, version 6.1, Small-Molecule Drug Discovery Suite 2013-3. Glide, version 6.1 2013.

52. Ritchie, D.W. Evaluation of protein docking predictions using Hex 3.1 in CAPRI rounds 1 and 2. Proteins 2003, 52, 98-106.

53. Taams, L.S.; Vukmanovic-Stejic, M.; Smith, J.; Dunne, P.J.; Fletcher, J.M.; Plunkett, F.J.; Ebeling, S.B.; Lombardi, G.; Rustin, M.H.; Bijlsma, J.W., et al. Antigen-specific T cell suppression by human CD4+CD25+ regulatory T cells. European journal of immunology 2002, 32, 1621-1630, doi:10.1002/1521-4141(200206)32:6<1621::AIDIMMU1621>3.0.CO;2-Q.

54. Maestro, v. 12.3.013, Schrödinger, LLC: New York, NY, 2020, 2020.

55. Frisch, M.J.; Trucks, G.W.; Schlegel, H.B.; Scuseria, G.E.; Robb, M.A.; Cheeseman, J.R.; Scalmani, G.; Barone, V.; Mennucci, B.; Petersson, G.A., et al. Gaussian 09, Gaussian, Inc., Wallingford CT, 2009), 2009.

56. Bella, J.; Liu, J.; Kramer, R.; Brodsky, B.; Berman, H.M. Conformational effects of Gly-X-Gly interruptions in the collagen triple helix. Journal of molecular biology 2006, 362, 298-311, doi:10.1016/j.jmb.2006.07.014.

57. Halgren, T. New method for fast and accurate binding-site identification and analysis. Chemical biology $\mathcal{E}$ drug design 2007, 69, 146-148, doi:10.1111/j.1747-0285.2007.00483.x.

58. Halgren, T.A.; Murphy, R.B.; Friesner, R.A.; Beard, H.S.; Frye, L.L.; Pollard, W.T.; Banks, J.L. Glide: A new approach for rapid, accurate docking and scoring. 2. Enrichment factors in database screening. J Med Chem 2004, 47, 1750-1759, doi:Doi 10.1021/Jm030644s. 
59. Friesner, R.A.; Banks, J.L.; Murphy, R.B.; Halgren, T.A.; Klicic, J.J.; Mainz, D.T.; Repasky, M.P.; Knoll, E.H.; Shelley, M.; Perry, J.K., et al. Glide: A new approach for rapid, accurate docking and scoring. 1. Method and assessment of docking accuracy. J Med Chem 2004, 47, 1739-1749, doi:Doi 10.1021/Jm0306430.

60. Friesner, R.A.; Murphy, R.B.; Repasky, M.P.; Frye, L.L.; Greenwood, J.R.; Halgren, T.A.; Sanschagrin, P.C.; Mainz, D.T. Extra precision glide: docking and scoring incorporating a model of hydrophobic enclosure for protein-ligand complexes. J Med Chem 2006, 49, 6177-6196, doi:10.1021/jm051256o.

61. Bowers, K.J.; Sacerdoti, F.D.; Salmon, J.K.; Shan, Y.; Shaw, D.E.; Chow, E.; Xu, H.; Dror, R.O.; Eastwood, M.P.; Gregersen, B.A., et al. Molecular dynamics---Scalable algorithms for molecular dynamics simulations on commodity clusters. In Proceedings of Proceedings of the 2006 ACM/IEEE conference on Supercomputing - SC '06.

62. Jorgensen, W.L.; Tirado-Rives, J. The OPLS [optimized potentials for liquid simulations] potential functions for proteins, energy minimizations for crystals of cyclic peptides and crambin. J. Am. Chem. Soc. 1988, 110, 16571666, doi:10.1021/ja00214a001.

63. Jorgensen, W.L.; Tirado-Rives, J. Potential energy functions for atomic-level simulations of water and organic and biomolecular systems. Proceedings of the National Academy of Sciences of the United States of America 2005, 102, 6665-6670, doi:10.1073/pnas.0408037102.

64. Berendsen, H.J.C.; Grigera, J.R.; Straatsma, T.P. The Missing Term in Effective Pair Potentials. J Phys Chem 1987, 91, 6269-6271, doi:Doi 10.1021/J100308a038.

65. Salentin, S.; Schreiber, S.; Haupt, V.J.; Adasme, M.F.; Schroeder, M. PLIP: fully automated protein-ligand interaction profiler. Nucleic acids research 2015, 43, W443-447, doi:10.1093/nar/gkv315. 